\title{
A Framework of Space-Time Continuous Models for Algorithm Design in Swarm Robotics
}

\author{
Heiko Hamann and Heinz Wörn \\ Institute for Process Control and Robotics, \\ Universität Karlsruhe (TH), Karlsruhe, Germany \\ e-mail:\{hamann, woern\}@ira.uka.de
}

\begin{abstract}
Designing and analyzing self-organizing systems such as robotic swarms is a challenging task even though we have complete knowledge about the robot's interior. It is difficult to determine the individual robot's behavior based on the swarm behavior and vice versa due to the high number of agent-agent interactions. A step towards a solution of this problem is the development of appropriate models, which accurately predict the swarm behavior based on a specified control algorithm. Such models would reduce the necessary number of time-consuming simulations and experiments during the design process of an algorithm. In this paper we propose a model with focus on an explicit representation of space because the effectiveness of many swarm robotic scenarios depends on spatial inhomogeneity. We use methods of statistical physics to address spatiality. Starting from a description of a single robot we derive an abstract model of swarm motion. The model is then extended to a generic model framework of communicating robots. In two examples we validate models against simulation results. Our experience shows that qualitative correctness is easily achieved while quantitative correctness is disproportionately more difficult but still possible.
\end{abstract}

\section{Introduction}

The design and analysis of artificial self-organizing systems is a challenging task because these systems typically behave non-intuitively and no widely-accepted theory of self-organizing systems exists. One challenge in understanding self-organizing system is the micro-macro linkdetermining the relationship between global and local behavior patterns and vice versa (Schillo et al., 2000; Yamins, 2005). The problem of linking the micro- and macro-level applies also to the design of swarm robotic systems. Typically, a certain task is given and a control algorithm has to be designed to run on a single robot; this corresponds to solving the problem of the micro-macro link. Currently, the use of simulations or even experiments for design support is a common but not satisfying solution, as it is time consuming and the results are most likely suboptimal. This difficulty might be overcome by appropriate models providing fast answers to questions asking for key parameters or overall performance based on first drafts of a control algorithm. In the following sections, we discuss the challenges in modeling multi-agent and complex systems as well as the state-of-the-art knowledge.

\subsection{Modeling Robotic Swarms}

A good motivation for the modeling approach in agent systems is given by Schweitzer (2003): 
To gain insight into the interplay between microscopic interactions and macroscopic features, it is important to find a level of description that, on the one hand, considers specific features of the system and is suitable for reflecting the origination of new qualities, but, on the other hand, is not flooded with microscopic details. In this respect, agent models have become a very promising tool mainly for simulating complex systems. A commonly accepted theory of agent systems that also allows analytical investigations is, however, still pending because of the diversity of the various models invented for particular applications.

This necessary reduction of microscopic details can most likely only be achieved by a probabilistic approach and through abstraction. The exact prediction of a robot's position is not only ruled out by the chaos-theoretic argument, stating that the initial setting can never be measured with sufficient accuracy, but also by involved processes being widely considered to be probabilistic in principle, e.g. wireless communication or noise in sensory information. Furthermore, significant sources of complexity are the agent-agent interactions. This complexity can be overcome by omitting an explicit agent representation.

\subsection{Related Work}

Research in agent-based modeling dates back to the self-reproducing automata of Neumann (1966) that, due to limited computational resources, was unpopular until its breakthrough in form of the new field of artificial life founded by Langton (1989). Since then, agentbased modeling has been applied to many fields ranging from logistics to social networks. It may appear obvious to use agent models from biology in swarm robotics; however, models of robotic swarms differ from biological or sociological models in their synthesis. In the case of swarm robotics, an extensive knowledge of the agents' interior exists because they have been constructed. In addition, robots typically have the ability to communicate exact data via electromagnetic radiation, which distinguishes them from the majority of biological models. Nevertheless, biological models might become more relevant, if minimalist hardware is applied, and an extensive progress has been achieved in the field of mathematical biology (Okubo, 1986; Grünbaum and Okubo, 1994; Okubo and Levin, 2001; Edelstein-Keshet, 2006; Mogilner and Edelstein-Keshet, 1999). Okubo (1986) provides a good review of a variety of swarm modeling concepts. Typically, the macroscopic approaches are phenomenological, that is, a macroscopic description is derived based on observations of the swarm behavior. However, "many diverse rules can lead to aggregates that look and behave quite similarly" as discussed by Edelstein-Keshet (2006). The microscopic approaches are based on simulations and verified by observing the resulting macroscopic behavior. This approach is in contrast to what we pursue in our work. We design the local rules ourselves and derive a macroscopic model with a strong connection to the microscopic level.

In swarm robotics there is only a small variety in mature state-of-the-art models ready to support the algorithm design phase despite great efforts lasting for almost a decade. Martinoli (1999) presents a modeling technique based on rate equations which was successfully applied to several scenarios. These rates quantify fractions of the swarm being in the same state. The temporal evolution of such a rate is, in most cases, easily derived and typically based on geometric considerations. For more information about the rate equation approach, see (Martinoli et al., 2004; Lerman et al., 2005). A methodologically very sophisticated approach is reported in (Correll and Martinoli, 2006; Correll, 2007) where parameters of the rate equations are determined by using system identification techniques. 
A drawback of these rate equation approaches is the limited representation of space - either homogeneous space is assumed or a rough discretization by states is done. Galstyan et al. (2005) present a generalization of the beforehand applied type of Master Equation (i.e., the foundation of the rate-equation approaches) for robot densities in continuous space. Similar to the biological models referenced above, this work is also a phenomenological macroscopic approach without the establishment of a connection to the microscopic level. Furthermore, no comparison to simulations is presented.

Many swarm robotic scenarios are based on heterogeneous space, for example, aggregation, exploration of non-periodic environments, transport, and collective movement. In these applications, an explicit representation of space in the model is helpful, if not necessary. Such models can be found in physics describing the motion of many entities or particles in continuous space. Typically, in physics there are microscopic as well as macroscopic models available for the same phenomenon, for example kinetic theory (microscopic) and thermodynamics (macroscopic). The simplification of treating a robot as a physical particle is adequate at least for extensive robotic swarms. This means, essentially, reducing the geometry of a robot to a cross section.

This approach of using models from physics is still somewhat related to the rate equation approach due to the application of numerical methods: The equations provided by physics are often not analytically solvable. Therefore, methods of numerical analysis have to be applied. In turn, this leads back to discrete representations due to the limitations of our computers. Finally, the equations effective in each point of the discretized space might be understood as rate equations of swarm fractions entering and leaving the associated area. This discretization can, however, be refined arbitrarily when provided with enough resources (computational power and memory).

In addition to the rate equation model, several other preliminary or rather specialized models were proposed: Winfield et al. (2005) present a promising formal method using temporal logic to specify and possibly prove emergent swarm behavior. Soysal and Şahin (2007) analytically derive a model for an aggregation behavior by applying combinatorics and linear algebra. Bjerknes et al. (2007) present a model of the collective motion algorithm reported by Nembrini et al. (2002) which is discussed in this paper as well. Compared to its inherent expressiveness, their model is surprisingly simple and mainly based on measured agent-agent interaction frequencies and durations.

In our model, we decided to follow the modeling approaches originating in physics. Based on Brownian motion, Schweitzer (2003) builds up the theory of Brownian agents, that is, self-propelled active particles. Similar approaches are those proposed in (Helbing et al., 1997; Vicsek et al., 1995) as well as a related recent work by Hogg (2006). In all three papers, the authors discuss the control of microscopic robots by Brownian motion, gradient ascent, and cooperation. While Hogg (2006) presents a phenomenologically motivated model, Schweitzer (2003) and Vicsek et al. (1995) intensively use microscopic simulations instead of macroscopic models. Helbing et al. (1997) study macroscopic models for a special scenario derived out of a microscopic description. However, these are sophisticated derivations adapted to the considered special scenario and a generalization of this approach seems to be improbable. In addition, all of these approaches except Hogg's lack direct agent-agent communication.

There are also many studies that apply control theory to model and control robotic swarms, e.g. see (Gazi and Passino, 2003; Feddema et al., 2002). We consider the preconditions for these approaches to be very limited. Full synchronization, global communication or communication to a fixed group of neighbors and deterministic behavior are often assumed. On the 
one hand, these approaches allow extensive stability analysis and certainties. On the other hand, the nondeterministic component, strictly local communication, and the possibility of coherence breakdowns are very relevant in swarm robotics and are not addressed in these control theoretic approaches.

In the next section, a short overview of Brownian motion and the variety of its applications is provided.

\subsection{Brownian Motion, the Langevin Equation, and the Fokker-Planck Equation}

Brownian motion is named in the honor of Robert Brown. Brown (1828) reported his observations of particles within the vacuoles of pollen grains under the microscope. He observed the now well-known random motion of a particle bigger by one order of magnitude compared to the size of the molecules in which it floats. The phenomenon of Brownian motion was mathematically solved by Einstein (1905) in one of his Annus Mirabilis papers and independently by Smoluchowski (1906). Three years later, Langevin (1908) discovered a different and easier approach with the microscopic description of Brownian motion - citing from (Lemons and Gythiel, 1997, a commented translation of Langevin's paper):

While Einstein, starting from reasonable hypotheses, derived and solved a partial differential equation [...] governing the time evolution of the probability density of a Brownian particle, Langevin applied Newton's second law to a representative Brownian particle. In this way, Langevin invented the $F=m a$ of stochastic physics now called the Langevin equation.

Fokker (1914) and Planck (1917) then analyzed the probability distribution of the velocity in the diffusion process by deriving a partial differential equation now called Fokker-Planck equation - a macroscopic description. However, this was only the first use of this equation which has an extensive variety of applications. This is also indicated by the independent rediscovery of this equation by Kolmogorov (1931). In his honor it is called the Kolmogorov forward equation describing time- and state-continuous Markov processes. Later, it was shown that the Fokker-Planck equation can also be applied to systems far from thermodynamic equilibrium. It is used to describe noise in quantum optics, for example, in modeling a laser (Risken, 1984) which can also be considered a self-organizing system as investigated in synergetics founded by Haken (1977). Schweitzer (2003) developed the concept of Brownian agents which are active particles with an internal energy depot and self-driven motion. For such reactive agents a generalized Langevin equation can be derived to describe the agents activities and offers possibilities of analysis including the formal derivation of a Fokker-Planck equation.

Our work is based on Schweitzer's concept of Brownian agents. We then extend it to a model of communicating robots, and compare it to results of two simulations.

\section{Modeling Robot Motion}

In swarm robotics, the motion of a robot is typically categorized in two types: First, the robot performs a type of random walk. Second, it moves in a direction determined by processing information provided by the environment. In general, there are three causes for random 


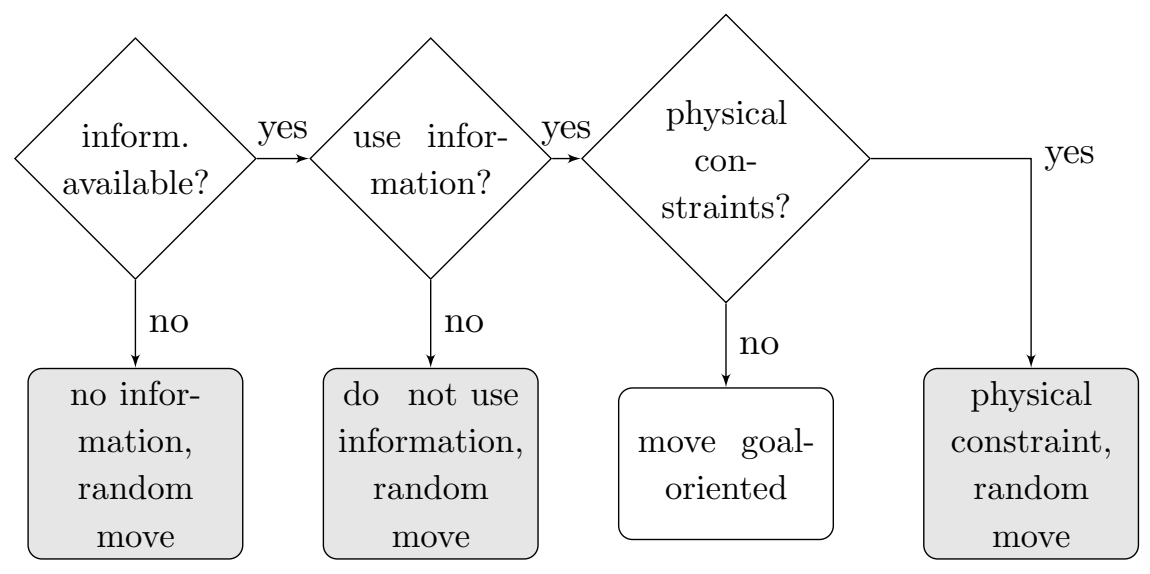

Figure 1: Schematic, classes of random moves.

motion (cf. Fig. 1): One, there is no information available to the robot; thus, the robot has to move randomly. Two, information is available but the autonomous robot decides to move randomly instead of exploiting this information. Three, information is available and the robot would like to move goal-oriented but due to physical constraints, for example, another robot blocking its way, a random direction needs to be chosen to keep moving and to avoid deadlocks.

We present a macroscopic model based on the Fokker-Planck equation derived from a microscopic model, the Langevin equation. Both the Langevin equation and the Fokker-Planck equation incorporate both types of motion, that is, random and goal-oriented motion. In this section we do not discuss the source of information available to a robot helping to determine its goal-oriented motion - this will be explored in the next section.

We assume that our robots move at nominal speed at all times and, therefore, disregard acceleration. This is a reasonable simplification, as the inertia being effective in the typical swarm robot is rather small compared to the engine power which results in acceleration times below one second. The motion of such a single Brownian agent is described by a Langevin equation of its position $\mathbf{R}$

$$
\dot{\mathbf{R}}(t)=-\mathbf{A}(\mathbf{R}(t), t)+B(\mathbf{R}(t), t) \mathbf{F}(t)
$$

with a random dislocation $\mathbf{F}$ due to fluctuating forces. Function $\mathbf{A}$ is a direction and describes the deterministic motion based on information provided by the environment (also information indirectly provided by other agents). Function $B$ describes the random motion. $\mathbf{A}$ and $B$ are characterized by the underlying control algorithm as discussed later. Using a computer and a random number generator the motion of such an agent can be simulated. The result is one trajectory out of the infinite set of all potential trajectories. However, to deduce the performance of a control algorithm, we are interested in the average behavior of the swarm. This behavior could be identified by sampling many trajectories, for example, by Monte Carlo simulations of the Langevin equation for a number of coexisting agents. However, this approach is microscopic and suffers from the same problems as standard robotic simulations, such as high computational cost.

From this microscopic description, a macroscopic description can be derived (as discussed below) - the Fokker-Planck equation 


$$
\frac{\partial \rho(\mathbf{r}, t)}{\partial t}=-\nabla(\mathbf{A}(\mathbf{r}, t) \rho(\mathbf{r}, t))+\frac{1}{2} Q \nabla^{2}\left(B^{2}(\mathbf{r}, t) \rho(\mathbf{r}, t)\right),
$$

where $Q$ is the displacement by a collision. $\rho(\mathbf{r}, t) d r_{x} d r_{y}$ is the probability of encountering a particle at position $\mathbf{r}$ within the rectangle defined by $d r_{x}$ and $d r_{y}$ at time $t$. It should be noted that we use $\mathbf{R}$ for positions of individual robots and $\mathbf{r}$ for points in space not directly connected to an explicit robot. Equations 1 and 2 are the two cornerstones of our model framework which will be used throughout this paper. We restrict ourselves to the two-dimensional case, but the step to a three-dimensional model is straightforward.

The Fokker-Planck equation implements the necessary abstraction as described above and treats rapidly-changing parameters as noise. The equation is still exact if this noise is generated by a Gaussian process, that is, it is fully determined by the first two moments. It gives the temporal evolution of the probability density describing the position of the agent.

The derivation of the Fokker-Planck equation for a given Langevin equation offers the possibility of analytically deriving some macroscopic aspects of a robot swarm out of a microscopic description. This derivation is a classical piece of statistical mechanics (among other fields), although, for the special case discussed here, such derivations are quite rare in literature. Similar derivations can be found in (van Kampen, 1981; Haken, 1977; Doob, 1953). A derivation following Haken (1977) is given in the appendix.

After having obtained the specific Fokker-Planck equation, we want to solve it. This is a non-trivial undertaking even with the help of standard commercial mathematical tools. The easiest way of solving the discretized equation is the forward integration in time. However, more sophisticated methods are needed to speed up the process and to maintain stability.

The main features of the agents' movements are captured by eq. 2 and 1 . In order to model a swarm of communicating robots, essential extensions have to be made. Appropriate functions A and $B$ need to be defined. These have to be coupled with equations that model the processes taking place in the environment.

\section{Modeling Agent-Agent Interactions}

In the preceding section we did not discuss the origin of the information which enables a robot to move goal-oriented. This origin could be a physically defined gradient, e.g. heat or natural gas, which is relatively easy to model. However, in applications of swarm robotics there is typically some form of cooperation making communication necessary. Thus, the modeling framework based on the Fokker-Planck equation needs to be extended to provide a way of modeling communication between robots. Because in physics there is no model of particles which communicate following a defined protocol, we have to drop the particle analogy in this section. However, the standard equations (eq. 1 and 2) remain as the matrix of our model because they correctly describe the fundamental effects of motion independent of external information determining the robot's goal-oriented movements. First, we discuss how an external influence to the robot's motion (that might be influenced by the swarm as well) can be modeled in general. Second, we explain why modeling direct communication is a challenging task. 


\subsection{External Influence}

Our general approach to incorporate external influence in the robot's movements is defining $\mathbf{A}(\mathbf{r}, t)$ as a function of a potential field $P$ describing features of the environment (e.g., magnetic fields or pheromones). In the following, the use of both mental models of function $\mathbf{A}$ as a component of the Langevin equation (eq. 1) or of the Fokker-Planck equation (eq. 2) will lead to the same results. For example, we can define $\mathbf{A}$ using the gradient of $P$

$$
\mathbf{A}(\mathbf{r}, t)=-\nabla P(\mathbf{r}, t) .
$$

By this definition we model a gradient ascent, that is, the robots aggregate at the closest maximum of $P$. Potential $P$ can be temporally and spatially variant and we can couple the temporal evolution of $P$ with the density $\rho$ (eq. 2 ). Thus, there is an extensive variety of possible applications: physically-defined processes (e.g., diffusion, heat flow, fluid dynamics, propagation of electromagnetic waves) but also heuristic approaches of possibly macroscopic effects (e.g., message propagation). Additionally, a discount coefficient should be included, especially in the case of a gradient ascent, making sure $\mathbf{A}$ drops with increasing density. Otherwise, the robots could aggregate in arbitrarily high densities.

The possibilities of defining a temporal evolution of $P$ are manifold. In the case of physically well-defined processes, this definition can be easy. For example, for a simple diffusion process we get

$$
\frac{\partial}{\partial t} P(\mathbf{r}, t)=D \nabla^{2} P(\mathbf{r}, t)
$$

for some diffusion constant $D$. Indirect communication (e.g., stigmergy) can be modeled by extending eq. 4 . In a simple case where robots permanently emit material that diffuses and evaporates thereafter (e.g., pheromones), an additive term proportional to the robot density and a subtractive term proportional to the potential are added to eq. 4; for example:

$$
\frac{\partial}{\partial t} P(\mathbf{r}, t)=D \nabla^{2} P(\mathbf{r}, t)+\gamma \rho(\mathbf{r}, t)-\theta P(\mathbf{r}, t),
$$

for constants $\gamma$ and $\theta$. The disposal of material could be space- and time-dependent leading to a function $\gamma(\mathbf{r}, t)$. As mentioned above, the possibilities are manifold and modeling them is quite intuitive.

Finding an appropriate model for more sophisticated processes such as the communication of messages (e.g., point-to-point, gossiping) is more problematic. This problem is discussed in the next section.

\subsection{Challenge of Modeling Communication}

The problems of modeling and simulating communicating robots are closely related to those experienced in the field of ad hoc and sensor networks. Most of the work in this field is based on simulations; for example, using ns-2 (McCanne et al., 1997). Analytic results are rare and the ones which exist are often only valid for special situations, such as the one-dimensional case. For example, this applies to the problem of determining the probability of a successful (multi-hop) message transfer between two points for a given density - called path probability $P$ (path) by Bettstetter (2004). He states: "Reviewing the literature on ad hoc networks, it seems that an analytical approach for calculating $P$ (path) does only exist for one dimension and an infinitely large system space. Since we are mainly interested in two-dimensional 
bounded areas, we approach the problem by means of simulation." Here, simulation means sampling random topologies in the tens of thousands for uniform distributions, not considering non-uniform distributions. Combined with a numerical solver of our model, this method is infeasible since we would either have to draw thousands of samples per time step or rely on statistically insignificant data. Therefore, we have to approximate the effects of communication heuristically, that is, macroscopically, and in closed form. Obviously, this approximation will be one of the main challenges while modeling scenarios which include communicating robots.

\section{Illustrative Applications}

The Fokker-Planck equation in the form as stated above is applicable to a variety of swarm robotic scenarios. It is mainly adapted by adjusting the functions $\mathbf{A}$ and $B$ to the given situation. In fact, this adaptation is another modeling step and, therefore, not trivial but rather a constructive step. Typically, $\mathbf{A}$ and $B$ cannot be derived based on microscopic factors only but have to be found by a mix of micro- and macroscopic influences. This procedure implies that the modeler has at least a fundamental understanding of the occurring macroscopic processes. Otherwise we would have solved a crucial part of the 'emergence problem' (Holland, 1998; Deguet et al., 2006).

In the following sections, we present two scenarios based on two different concepts of coordination and communication. The first one is a rather simple model and only qualitative in nature. We present it to demonstrate the ease of achieving qualitatively correct predictions with our model framework. The second example is a sophisticated model allowing quantitative conclusions. Preliminary results of this model were presented in (Hamann and Wörn, $2007 \mathrm{~b})$ where problems in handling the arising variance were identified. Here, we present an improved model.

\subsection{Emergent Taxis}

As a first example we give a qualitative model of the emergent taxis scenario as presented by Nembrini et al. (2002) and analyzed by Bjerknes et al. (2007). This algorithm is a great example of how a comparatively complex task, moving collectively towards a beacon, can be solved by a simplistic approach. In the algorithm there is no instruction telling a robot to move towards the beacon, which is possibly why it is labeled 'emergent'.

\subsubsection{Description of the Emergent Taxis Scenario}

The primary task of the robot swarm is to move towards a beacon. Secondary tasks are the avoidance of collisions and to preserve coherence, that is, to keep a connection with a predefined number of neighbors. All robots have a range-limited wireless communication device. Each robot frequently sends a ping and permanently counts the individual robots from which it receives such a ping. All robots move forward until one of the following three events occurs (cf. Fig. 2):

1. If two robots get too close to each other they have to avoid the collision.

2. Robots receiving pings from too few neighbors try to regain coherence. 


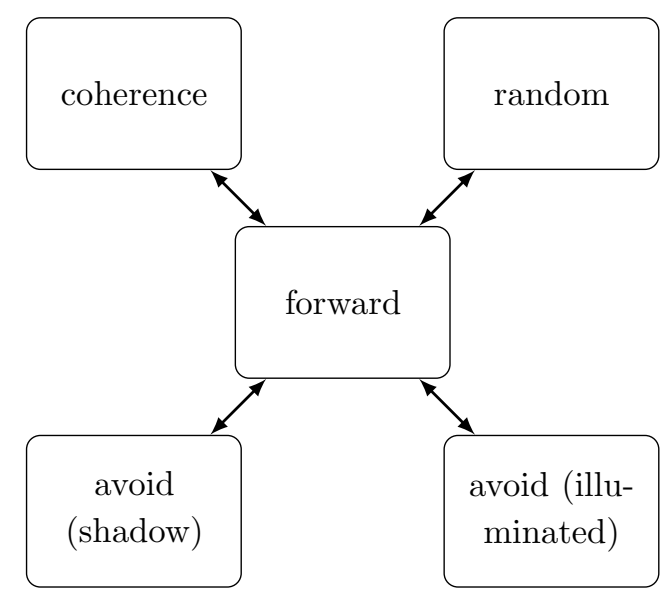

Figure 2: State diagram for the emergent taxis scenario, reproduced after (Bjerknes et al., 2007).

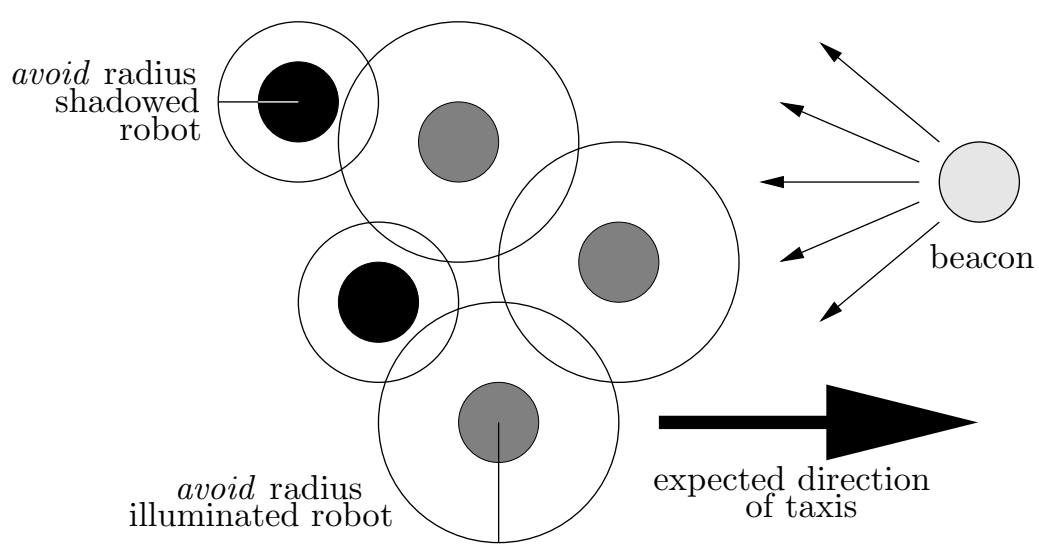

Figure 3: The emergent taxis scenario with five robots (black circles for shadowed and dark gray for illuminated robots) and a beacon to the right (light gray circle). Circles around the robots show the avoid radii. The communication range is not shown.

3. Robots having recently regained coherence change their direction randomly.

The following process ensures coherence: If the number of individual robots, from which a ping was recently received, drops below a threshold $\alpha$ (condition for the transition to the coherence state), the robot executes a U-turn and re-enters the forward state. The underlying assumption is that the robot was about to detach and lose connection to the swarm. The U-turn followed by moving forward will reconnect the robot. After the number has reached the threshold $\alpha$ again (condition for the transition to the random state), the robot makes a random turn and re-enters the forward state. In the simulations given by Bjerknes et al. (2007) $\alpha$ is always set to the swarm size $N=\alpha$. The two avoid states implement the collision avoidance behavior. In both states, the robots turn away from other robots which were getting too close. The difference of these states is discussed in the following.

Up to this point the algorithm triggers an aggregation behavior (ideally) ensuring that the swarm stays connected. To extend this behavior by a collective movement towards the beacon, 


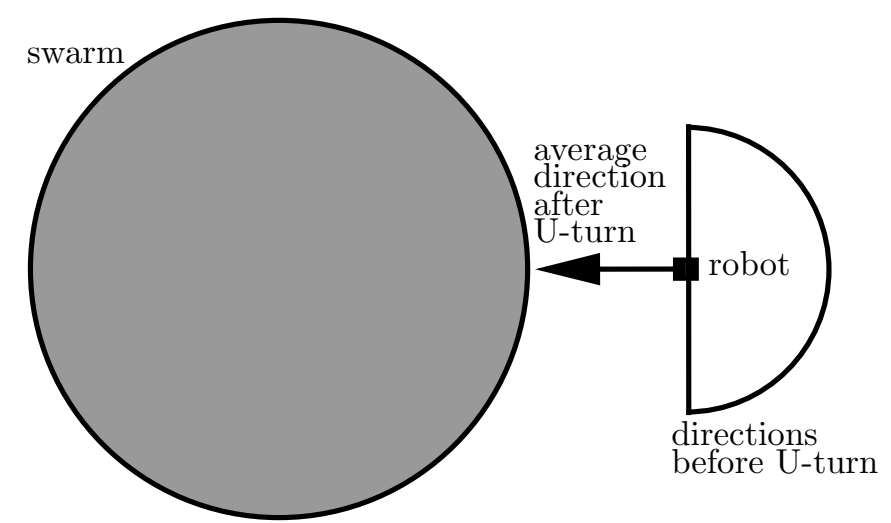

Figure 4: Schematic representation of a U-turning robot that was about to detach from the swarm, indicating the average direction towards the swarm's barycenter after the U-turn.

each robot is equipped with a long-range beacon sensor enabling it to sense whether there is a beacon. It is assumed that occlusions are possible: a robot, which is in the shadow of another, will not perceive the beacon (cf. Fig. 3). In the following, we distinguish two groups of robots: illuminated (perceiving the beacon) and shadowed (not perceiving the beacon). The crucial point of the emergent taxis algorithm is to define different avoid radii for these two groups. Illuminated robots have a bigger avoid radius than shadowed robots. This difference between the two avoid states is enough to determine the desired direction of the taxis.

\subsubsection{Derivation of the Model}

Now, we want to model the fundamental behavior. Two insights about the macroscopic process are helpful to obtain a simple model. First, we note the symmetry breaking: illuminated robots avoid shadowed robots but the reverse does not occur - as if shadowed robots would not perceive illuminated ones. Thus, the goal-oriented movements of illuminated robots (behavior implemented by the coherence state) will be interrupted more often than the movements of shadowed robots. This effect will result in a higher ratio of random motion for illuminated robots compared to shadowed robots. Furthermore, the asymmetric avoid radii result in a decreased number of collisions for shadowed robots. Stated differently, the effective robot density for shadowed robots will be lower then the actual robot density as it is perceived by and effective to illuminated robots. While illuminated robots have to avoid shadowed robots, shadowed robots will not avoid illuminated robots because the illuminated robots have to react earlier, that is, at bigger distances. Second, we assume that after having executed their U-turn, robots will, on average, head towards the barycenter of the swarm (see Fig. 4 showing a detaching robot).

Given these insights, the adaptation of the model framework to the emergent taxis scenario is straightforward. At first, we have to introduce the distinction between illuminated and shadowed robots. Considering the probabilistic nature of our model we introduce function $s(\mathbf{r}, t)$, giving the probability of a robot at position $\mathbf{r}$ and time $t$ of being shadowed. The values of $s$ are achieved by integrating over $\rho$ along beams originating in the beacon ( $\rho$ is a particle density, i.e., integrating over all space we get the swarm size $\left.N=\int \rho(\mathbf{r}, t) d \mathbf{r}\right)$. To ensure the probability property, we define a maximum of $s_{\max }=1$. We define for the general case 


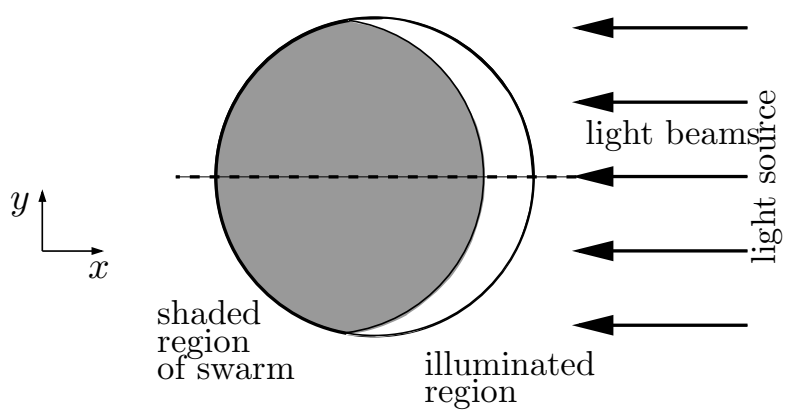

Figure 5: Schematic representation of the light model used. The light beams are assumed to be parallel. On the right hand side of the swarm a region with $s>0$ is found (illuminated region). On the left hand side there is a region with $s=0$. The actual size of the illuminated region depends on the swarm size, robot size/shape etc. Integration over $\rho$ along lines parallel to the dashed line determines $s$ (cf. eq. 6).

\begin{tabular}{|c|c|}
\hline Parameter & Value \\
\hline$N$ & 10 \\
$c_{1}$ & $50\left[1 / u^{2}\right]$ \\
$c_{2}$ & 7 \\
$c_{3}$ & 11 \\
$c_{4}$ & $50\left[1 / u^{2}\right]$ \\
$c_{5}$ & 23 (for $a_{1}$ ) and $30\left(\right.$ for $\left.a_{2}\right)$ \\
\hline
\end{tabular}

Table 1: Parameters used in the taxis scenario, by $u$ the unit length is indicated.

$$
s(\mathbf{r}, t)=\min \left(\int_{r_{y}^{\prime}=m r_{x}^{\prime}+k} \rho\left(\mathbf{r}^{\prime}, t\right) d \mathbf{r}^{\prime}, 1\right),
$$

with $\mathbf{r}^{\prime}=\left(r_{x}^{\prime}, r_{y}^{\prime}\right)^{T}$ and for a light source at $\mathbf{L}=\left(L_{x}, L_{y}\right)^{T}$. We define $m=\frac{r_{y}-L_{y}}{r_{x}-L_{x}}$ and $k=r_{y}-\frac{r_{y}-L_{y}}{r_{x}-L_{x}} r_{x}$ for $\mathbf{r}=\left(r_{x}, r_{y}\right)^{T}$. Here, we assume that the light source is far away in the $x$ direction. Thus, a simplified light model can be applied assuming the light beams arrive parallel to the $x$-axis (cf. Fig. 5). This simplifies eq. 6 to

$$
s(\mathbf{r}, t)=\min \left(\int_{r_{x}}^{\infty} \rho(\mathbf{r}, t) d \mathbf{r}^{\prime}, 1\right),
$$

for $L_{x} \rightarrow \infty$. An intuitive interpretation of this equation is to think of summing densities beginning at the light source backwards in the $x$ direction. If a point is reached, where the value sums up to one, we set $s=1$. Otherwise, $s$ is set to the sum reached thus far.

Based on $s$ we define the diffusion function $B$ as a sigmoid function

$$
B(\mathbf{r}(t), t)=\left(1+\exp \left(-c_{1} \rho(\mathbf{r}, t)+(1-s(\mathbf{r}, t)) c_{2}+s(\mathbf{r}, t) c_{3}\right)\right)^{-1},
$$

for constants $c_{1}, c_{2}$, and $c_{3}$ (see Table 1 for the values that were used in the following example). 


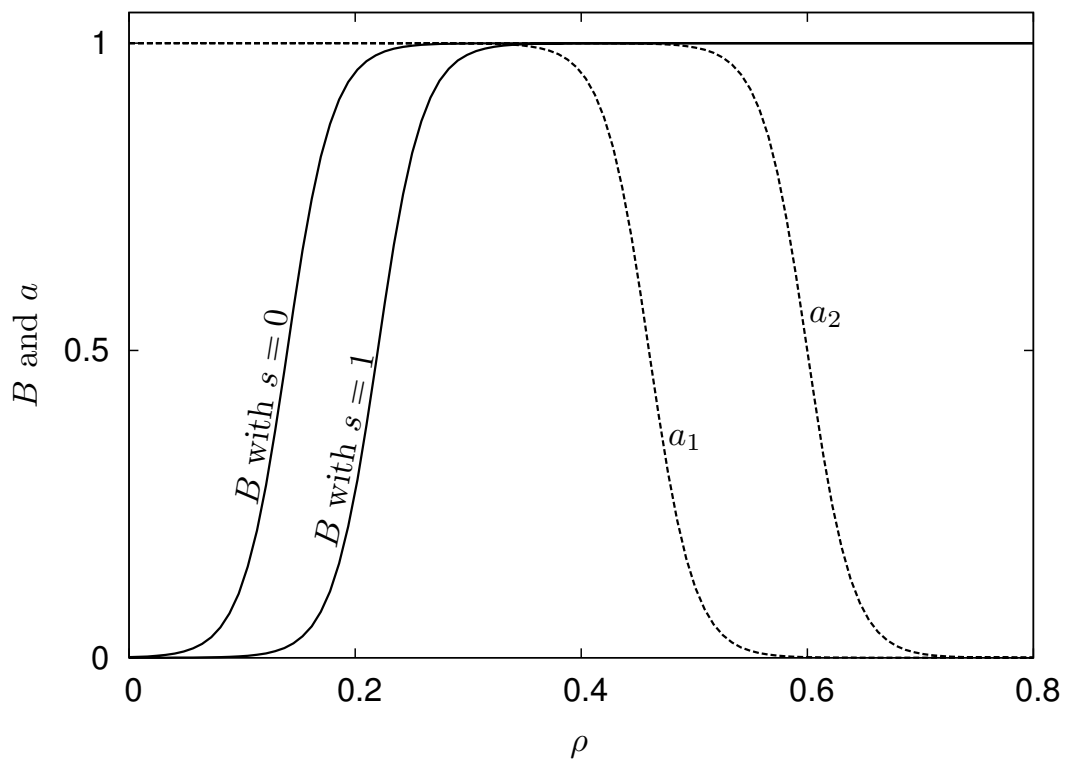

Figure 6: Examples for sigmoid functions $a$ and $B$ (for $s=0$ and $s=1$ ).

The gradient function $\mathbf{A}$ represents the threshold $\alpha$ and the direction of robots after having executed a U-turn. This representation is achieved by defining a potential $P(\mathbf{r}, t)$ which has a global maximum at the barycenter of the swarm at each time and whose gradient is normalized $\forall \mathbf{r}, t:\|\nabla P(\mathbf{r}, t)\|=1$. Combining this gradient with a sigmoid function which activates the U-turning behavior for low densities, we get

$$
\mathbf{A}(\mathbf{r}(t), t)=a(\mathbf{r}(t), t) \nabla P(\mathbf{r}, t),
$$

with

$$
a(\mathbf{r}(t), t)=\left(1-\left(1+\exp \left(-c_{4} \rho(\mathbf{r}(t), t)+c_{5}\right)\right)^{-1}\right),
$$

for constants $c_{4}$ and $c_{5}$. The parameters that were used in the following examples are given in Table 1. Plots of $B$ and $a$ are given in Fig. 6. It can be seen that the ratio of random movements $B$ is bigger for illuminated robots $(s=0)$ than for shadowed robots $(s=1)$ for a fixed density $\rho$ (for $0<\rho<0.35$ ). This difference implements the considerations about the effective density of shadowed robots stated at the beginning of this section.

\subsubsection{Results}

The obtained simple model is already capable of predicting the velocity of the swarm's collective motion in a qualitatively correct manner (change of the barycenter) as is shown in Fig. 7 which gives the distance covered by the swarm. The difference between the two runs is a shifted $a$-function $\left(a_{1}\right.$ and $\left.a_{2}\right)$. Higher swarm velocities are achieved by shifting $a$ to lower densities; as in this case robots can reach bigger distances from the barycenter (where the robot density gets lower) before they have to execute a U-turn. This effect corresponds to the observations by Bjerknes et al. (2007). They observed higher swarm velocities for bigger communication distances. Thus, our model's prediction is qualitatively in accordance with Bjerknes et al. (2007). 


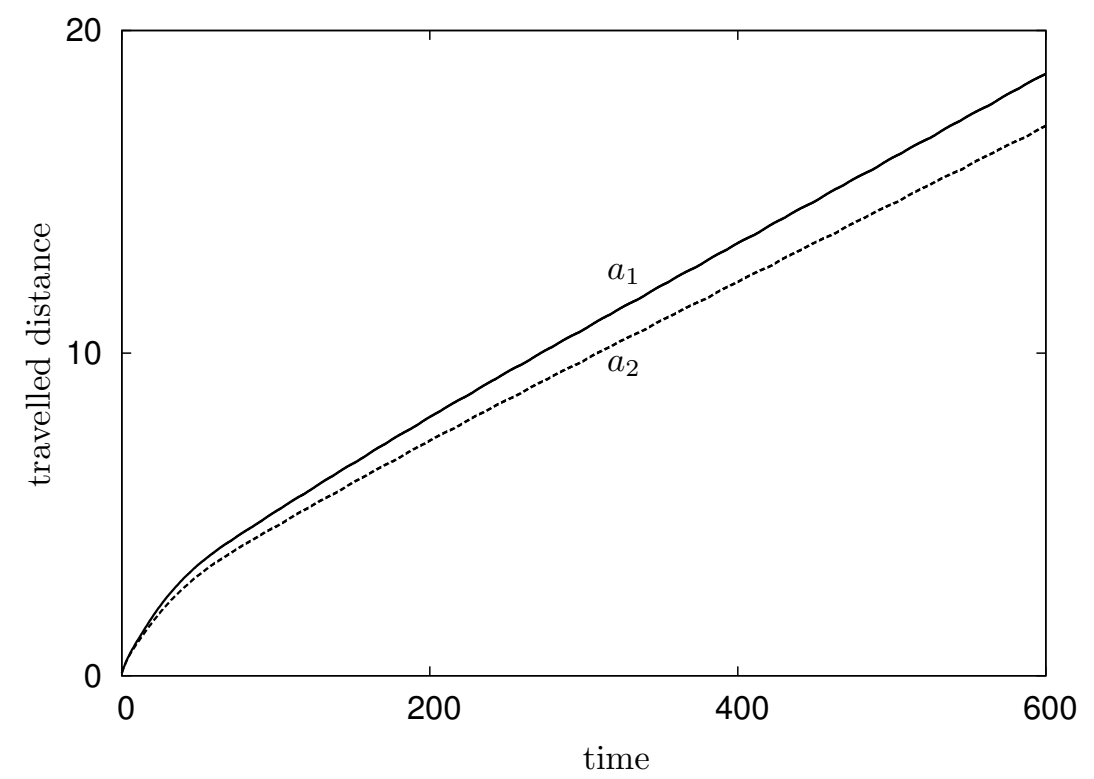

Figure 7: Distance covered by the swarm collectively measured on the basis of the barycenter for two variants of function $a$ (cf. Fig. 6).

\subsection{Collective Perception}

Collective perception is a process in which a group of autonomous agents collectively extracts the main features of an object or, more general, of the environment. These features can not be observed in principle or at least not within a reasonable time frame by a single agent. An algorithm inspired by honey bees implementing collective perception on a relatively low level of complexity was presented by Schmickl et al. (2007a). Their approach is called trophallaxis strategy in appreciation of the underlying behavior in social insects - e.g. mouth-to-mouth transfer of food in bees.

We give a short description of the scenario in the following section. The interested reader is referred to (Schmickl et al., 2007a; Schmickl and Crailsheim, 2006, 2008; Schmickl et al., 2007b,c) for more information, as well as (Hamann and Wörn, 2007b) where we report a preliminary model. Subsequently, we explain the trophallaxis strategy and give a derivation of the model.

\subsubsection{Description of the Trophallaxis Strategy}

The robots move in a bounded planar area. There are two designated areas of different sizes that we call 'target areas' in the following (cf. Fig. 8). The task of the swarm is to aggregate at the bigger target area. However, measuring the dimension of an area is beyond a single robot's abilities. Using the trophallaxis strategy even irregular areas, e.g. scattered and loosely connected areas, can be collectively perceived, although we restrict our investigations to simple circular shapes here. Many applications are possible using this behavior as a component, such as cleaning, monitoring, or transportation scenarios.

The simulated robots are able to communicate within a short range and with limited orientations (six communication devices mounted every $60^{\circ}$ around the robot, for more details 


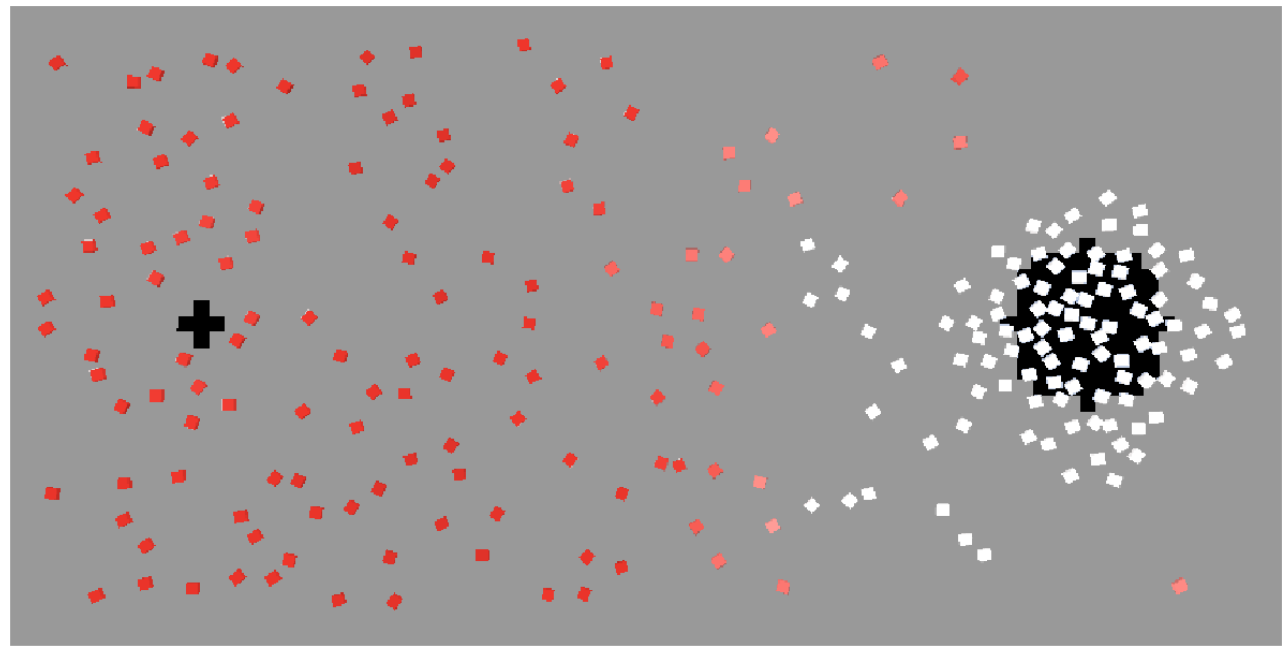

Figure 8: Screen-shot of the simulation showing two circular target areas of different size and cubic robots with their value of the potential field indicated by their brightness (the brighter, the higher the value).

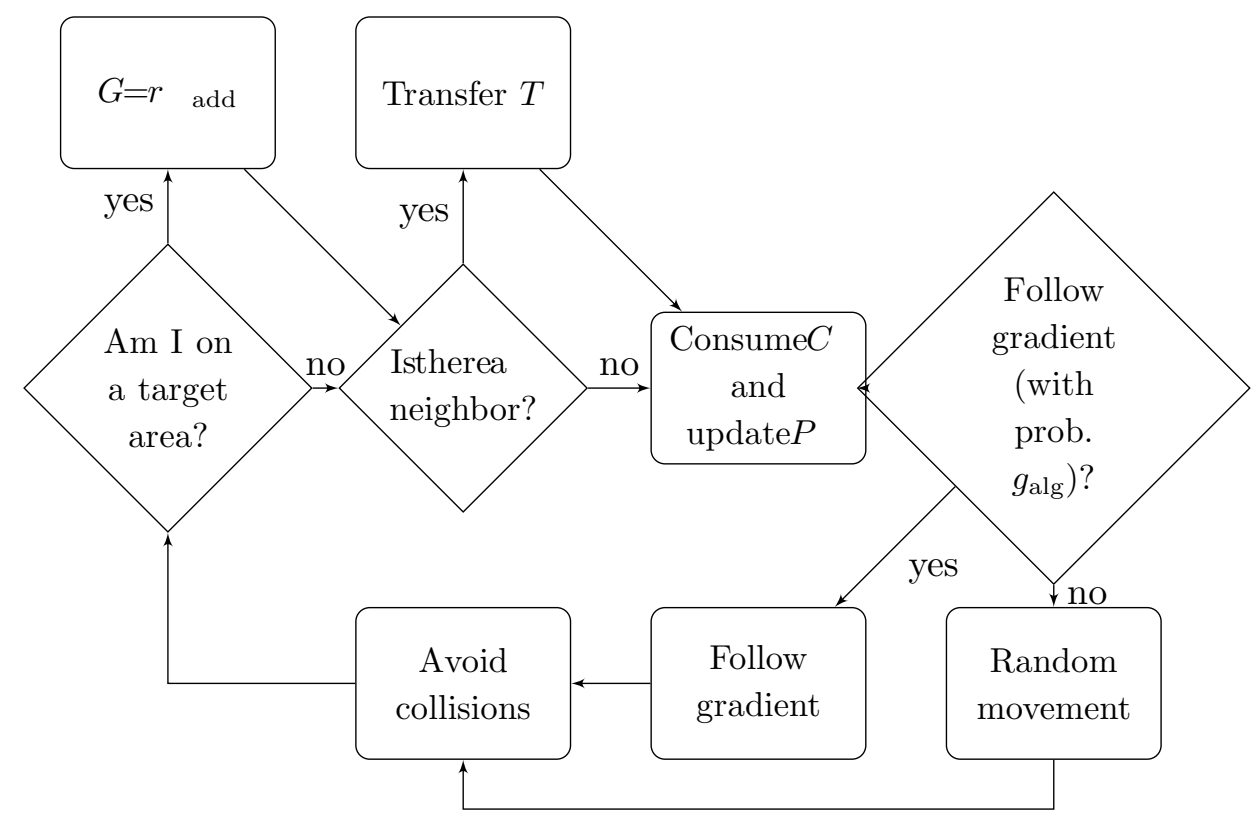

Figure 9: Schematic presentation of the trophallaxis control algorithm. The gathering $(G)$, transfer $(T)$, consumption $(C)$, potential field $P$, and the ratio of gradient ascent moves $\left(g_{\text {alg }}\right)$ are defined by eq. 11 to 15 . 
see Schmickl et al. (2007a)). As a side-effect of communication, an approximate bearing of the communication partner is determined. It is assumed that infrared devices are used for communication. Additionally, the robots are capable of perceiving certain features of the environment which enables them to determine whether they are on one of the target areas. The explicit feature and the corresponding sensor is not specified in (Schmickl et al., 2007a) and could be, for example, a light sensor or microphone depending on how the target area is marked.

The fundamental idea of the trophallaxis strategy is to build up a virtual potential field $P\left(\mathbf{R}_{i}, t\right)$ by assigning a value to each robot. This value is stored on each robot $i$ at position $\mathbf{R}_{i}$ and time $t$. If the swarm develops a potential field, with maxima at the target areas, robots can, in principle, be led to the target by a gradient ascent. This approach requires that a connection exists between the specific robot and the robots on the target area (via a low-level multi-hop communication, as described below). In the following paragraph we define an update rule that is applied by each robot to compute $P\left(\mathbf{R}_{i}, t\right)$.

If a robot $i$ is within a target area it adds an amount $r_{\text {add }}$ to its $P$-value (see Fig. 9 showing the simplified control algorithm). This addition to $P$ is defined by the amount $G$ that is potentially gathered by a robot per step

$$
G\left(\mathbf{R}_{i}, t\right)=\left\{\begin{array}{ll}
r_{\text {add }} & \text { if } \mathbf{R}_{i} \text { is within a target area } \\
0 & \text { else }
\end{array},\right.
$$

for addition rate $r_{\text {add }}$. See Table 2 for the values used in the following examples, if they are not explicitly stated. To homogenize the potential field a robot $i$ communicates with its neighbors $\mathcal{N}$ (robots within sensor range) and sends or receives a transfer amount $T$ that is proportional to the difference of the potential field values:

$$
T\left(\mathbf{R}_{i}, t\right)=\sum_{j \in \mathcal{N}}\left(P\left(\mathbf{R}_{i}, t\right)-P\left(\mathbf{R}_{j}, t\right)\right) r_{\text {transfer }},
$$

for the position of a neighbor $j \in \mathcal{N}$ at $\mathbf{R}_{j}$ being within communication distance $d_{\text {comDist }}$ of robot $i$ and transfer rate $r_{\text {transfer }}$. During each cycle a robot $i$ consumes an amount $C$ that is proportional to $P$. $C$ is the consumption amount which is defined by

$$
C\left(\mathbf{R}_{i}, t\right)=P\left(\mathbf{R}_{i}, t\right) r_{\text {consmpt }},
$$

for consumption rate $r_{\text {consmpt }}$.

The update rule for the potential field value of robot $i$ is defined by

$$
P\left(\mathbf{R}_{i}, t+\Delta t\right)=P\left(\mathbf{R}_{i}, t\right)+G\left(\mathbf{R}_{i}, t\right)+T\left(\mathbf{R}_{i}, t\right)-C\left(\mathbf{R}_{i}, t\right) .
$$

Before we define the actual rule which determines the robots' movements, we consider the two possible extremes of information being available to a robot in certain situations. In the case of an isolated robot, that is, the robot has no neighbors, and provided the robot has no knowledge about the past and stopping is not an option, all possible directions are equally useful. Thus, turning in a random direction and moving forward for some time to gain a maximum of exploration is a good strategy for this robot. Concerning the other extreme, a robot having neighbors with high values of $P$ at similar bearings, it is reasonable to move towards them in most of the cases. For all situations between these two extremes, an efficient 


\begin{tabular}{|l|c|}
\hline Parameter & Value \\
\hline average robot density $\bar{\rho}$ & $0.117188 / d^{2}$ \\
number of robots & 375 \\
arena dimensions & $80 d \times 40 d$ \\
target area radii & $1 d$ and $5 d$ \\
robot diameter & $1 d$ \\
communication distance $d_{\text {comDist }}$ & $3.5 d$ \\
avoiding distance $d_{\text {avoidDist }}$ & $0.75 d$ \\
robot speed & $0.25 d$ \\
\hline addition rate $r_{\text {add }}$ & $50 / \Delta t$ \\
consumption rate $r_{\text {consmpt }}$ & $0.01 / \Delta t$ \\
transfer rate $r_{\text {transfer }}$ & $0.5 / \Delta t$ \\
aggregation threshold $\delta_{\text {aggr }}$ & 0 \\
\hline
\end{tabular}

Table 2: Distances are given in robot diameters $d$ and time dependent values per simulation step $\Delta t$.

strategy is less obvious. The movements are described by three phases depending on $P\left(\mathbf{R}_{i}, t\right)$ as shown in Fig. 10. First, a robot moves completely at random if $P\left(\mathbf{R}_{i}, t\right)<\delta_{\text {aggr }}$, for an aggregation threshold $\delta_{\text {aggr }}$. Second, the ratio $g$ of gradient ascent movements to random movements increases linearly in $P$ for $\delta_{\text {aggr }}<P\left(\mathbf{R}_{i}, t\right)<\delta_{\text {sat }}$, for a saturation threshold $\delta_{\text {sat }}$. Third, this ratio $g$ is constant for $P>\delta_{\text {sat }}$. Now, we give a formal definition of function $g_{\text {alg }}$ defining the ratio of goal-oriented moves of a robot

$$
g_{\text {alg }}\left(\mathbf{R}_{i}, t\right)=\max \left(\min \left(\frac{P\left(\mathbf{R}_{i}, t\right)-\delta_{\text {aggr }}}{1000}, 0.75\right), 0\right) .
$$

The saturation threshold $\delta_{\text {sat }}$ is implicitly defined by $\delta_{\text {sat }}=750+\delta_{\text {aggr }}$. This approach ensures a minimal exploration rate of $25 \%$ independent of $P$ because the 'exploitation rate' $g_{\text {alg }}$ is limited to $75 \%$.

\subsubsection{Derivation of the Model}

Modeling this collective perception scenario with the Langevin and the Fokker-Planck equation (eq. 1 and 2) is mainly achieved by finding functions $\mathbf{A}$ and $B$ which describe the behavior well. Additionally, we need an equation which describes the temporal evolution of potential $P$. Defining these functions is a heuristic process and there is no unique solution.

Function A depends primarily on a vector representing the directional information, that is, the gradient $\nabla P$. We normalize this gradient because the robots are not influenced by the length of vector $\nabla P$ but by its direction only. The frequency of choosing the direction given by $\nabla P$ depends on the gradient ascent ratio $g_{\text {aggr }}$. We use an effective gradient ascent ratio $g_{\text {mod }}$ differing from $g_{\text {alg }}$ due to macroscopic effects as described later. Furthermore, we have to define a maximal density, otherwise the whole swarm could aggregate in one point. This can be incorporated in a discount coefficient $d(\rho) \in[0,1]$ with $d(0)=1$ and $d\left(\rho_{\max }\right)=0$. By $d$ the increasing difficulty of executing gradient ascent movements with increasing density is modeled. High densities result in less successful executions of gradient ascent moves because more collision avoidance actions are necessary. Finally, we multiply with the nominal velocity $v$. In terms of the Fokker-Planck equation this means the robot flow depends linearly on the 


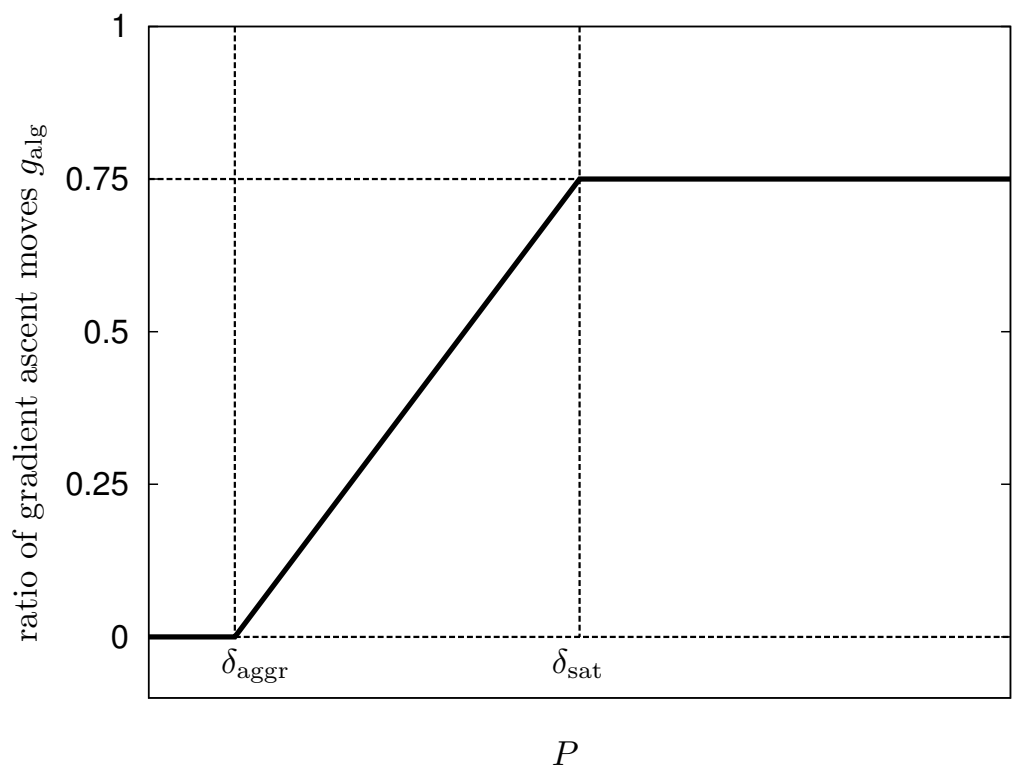

Figure 10: The ratio of gradient ascent moves $g_{\text {alg }}$ depending on potential $P$ as defined by the algorithm. For $P<\delta_{\text {aggr }}$ all moves are random, for $\delta_{\text {aggr }}<P<\delta_{\text {sat }}$ the probability of following the gradient increases linearly in $P$, and for $P>\delta_{\text {sat }}$ a robot follows the gradient in $75 \%$ of all moves.

velocity. In terms of the Langevin equation: The robot's displacement in direction $\nabla P$ is a fraction of $v$. Thus, we get

$$
\mathbf{A}=d(\rho) g_{\text {mod }}(P) \frac{\nabla P}{\|\nabla P\|} v
$$

Function $B$ describes the non-deterministic motion. Random movements are either caused by intended exploration with a rate of $\left(1-g_{\text {mod }}\right)$ or by a gradient ascent attempt disturbed by collision avoidance with a rate of $(1-d) g_{\text {mod }}$. Additionally, we have to model a slowdown of the robot's random movements. The spatial displacement caused by collision avoidance decreases with increasing density although the number of collisions increases as well. We can reuse $d$ as a good description of the normalized spatial displacement since the displacement is inversely proportional to the density (analogous to the mean free path in physics). Thus, multiplying by $d$ and velocity $v$ we get

$$
B=\left(\left(1-g_{\text {mod }}\right)+(1-d) g_{\text {mod }}\right) v d=\left(1-g_{\text {mod }} d\right) v d .
$$

The potential $P$ which represents the expected value for each point and for each time is modeled as in our earlier work (Hamann and Wörn, 2007b). We adopt two simplifications. First, the communication between the robots, which implements the homogenization of potential $P$, is considered to be a diffusion process. Second, this propagation of the potential field values is independent of the robot density, thus we assume total connectivity. This rather strong simplification is compensated by our method of measuring $g_{\text {mod }}$ as described below. $P$ is defined by 


$$
\begin{aligned}
\frac{\partial}{\partial t} P(\mathbf{r}, t)= & c_{\text {transfer }} \nabla^{2} P(\mathbf{r}, t) \\
& -r_{\text {consmpt }} P(\mathbf{r}, t) \rho(\mathbf{r}, t) \\
& +\delta_{\text {onTarget }}(\mathbf{r}, t) r_{\text {add }} \rho(\mathbf{r}, t),
\end{aligned}
$$

with

$$
\delta_{\text {onTarget }}(\mathbf{r}, t)= \begin{cases}1 & \text { if } \mathbf{r} \text { is within a target area at time } t \\ 0 & \text { else }\end{cases}
$$

and $c_{\text {transfer }}$ is obtained computationally

$$
c_{\text {transfer }}=r_{\text {transfer }}^{1 / d_{\text {compist }}} .
$$

With this definition, we ensure the effective coefficient for distance $d_{\text {comDist }}$ to be the transfer rate $r_{\text {transfer. }}$. We explain next how we determined the parameters of this model.

\subsubsection{Measured and Free Parameters}

The discount coefficient function $d(\rho)$ can be measured by counting the number of collisionavoiding actions of a robot group in an otherwise empty arena. This function's type (negative exponential) is easily diagnosed. However, it is difficult to determine the function quantitatively. In the control algorithm, virtual physics is used to avoid collisions, that is, the deflection is highly variable, and therefore, the difference between collision avoidance and regular moves is blurred. We decide to consider a deflection of at least $\pm 20^{\circ}$ as an avoidance move. $d$ is the ratio of such avoidance moves to the total number of moves. We obtained $d(\rho)=(1-\rho)^{3.7}$ as a good fit for the measurements, see Fig. 11. Consequently, we have reduced the complex and microscopic underlying processes to a single parameter of little expressiveness. However, this abstraction of microscopic processes is necessary and we readily accept it because it is measurable.

The effective gradient ascent ratio $g_{\text {mod }}$ is also based on measurements. We measured mean potential field values and corresponding mean values $g_{\text {eff }}$ for 13 histogram bins on the interval $g_{\text {eff }} \in[0,1200]$ over 600 simulation runs of 250 time steps each, see Fig. 12. In doing so, we use internal information, something that is impossible in biological systems, that is, that cannot be determined based on the observation of the behavior. We get a good measure for the frequency of goal-oriented actions as a function of $P$. Using $g_{\text {mod }}$, which is an approximation for $g_{\text {eff }}$, we include the high variance of $P$ in the model. Thereby, the simplification of total connectivity is compensated as well because this variance is mainly caused by alternating between connection and disconnection of robot groups. In addition, it turns out that the influence of the aggregation threshold $\delta_{\text {aggr }}$ creates a shift only allowing for the generalization from $g_{\text {eff }}$ to any $\delta_{\text {aggr }}$.

Although several parameters can be measured, some free parameters remain due to our empirical approach of the communication model. While the gathered potential field value in the simulation is just distributed among the robots, it is dispersed over the whole area in our model. Therefore, we scale the height of the potential field by a free coefficient. Also, the absolute displacement caused by a collision ( $Q$ in eq. 2$)$ cannot be measured because one avoidance event cannot be distinguished from the next one. The scaling parameter and $Q$ 


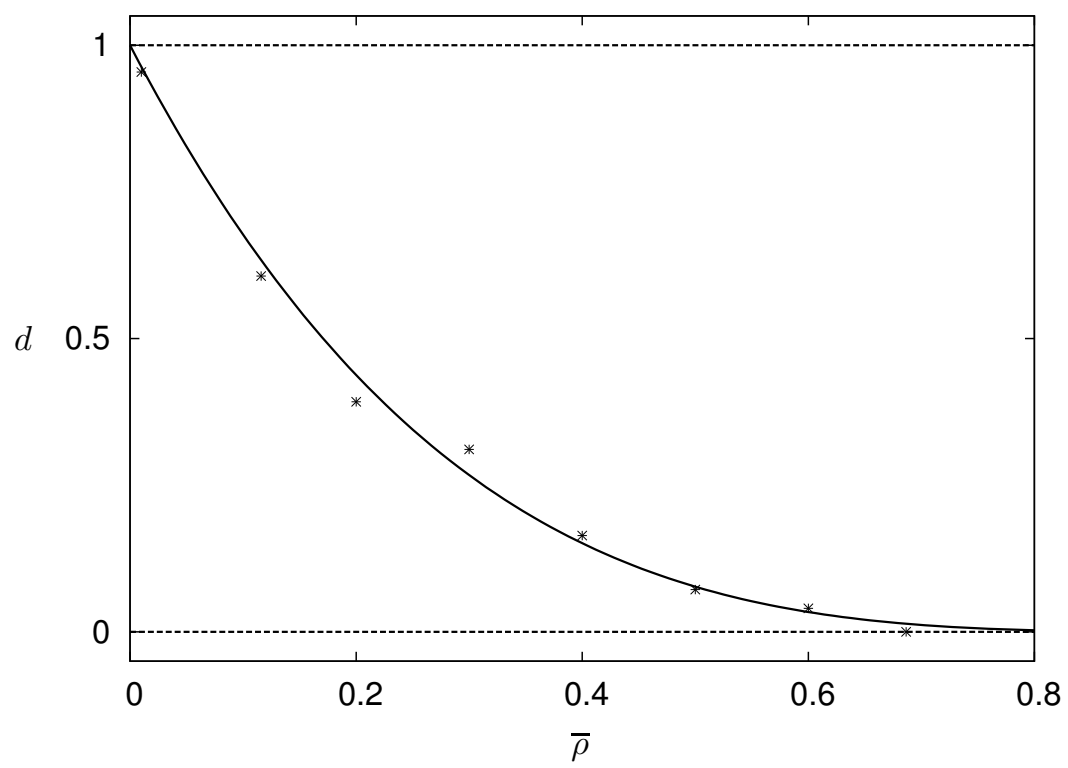

Figure 11: Discount coefficient $d$, asterisks show measured values (confidence intervals are within the symbols' sizes).

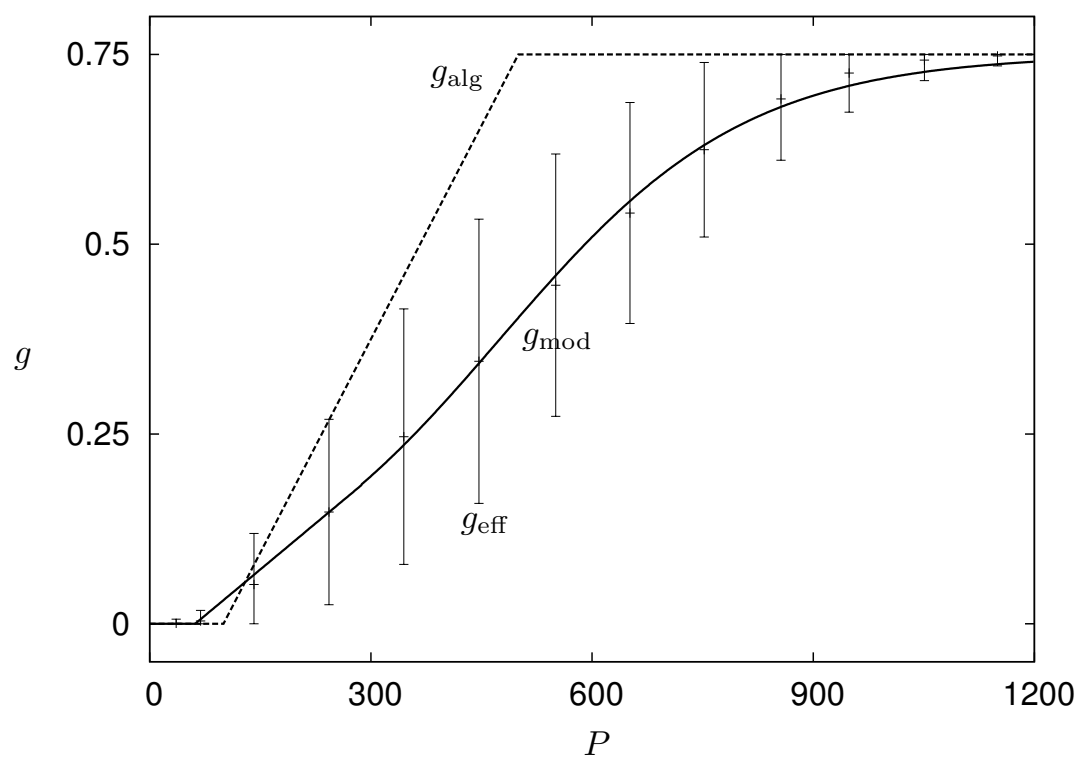

Figure 12: Ratio of gradient ascent moves $g_{\text {mod }}$ (solid line) for $\delta_{\text {aggr }}=100$ depending on potential $P$ as used in the model in comparison to measured values $g_{\text {eff }}$ (error bars show the standard deviation) and to $g_{\text {alg }}$ (dashed line) as defined in the algorithm. 


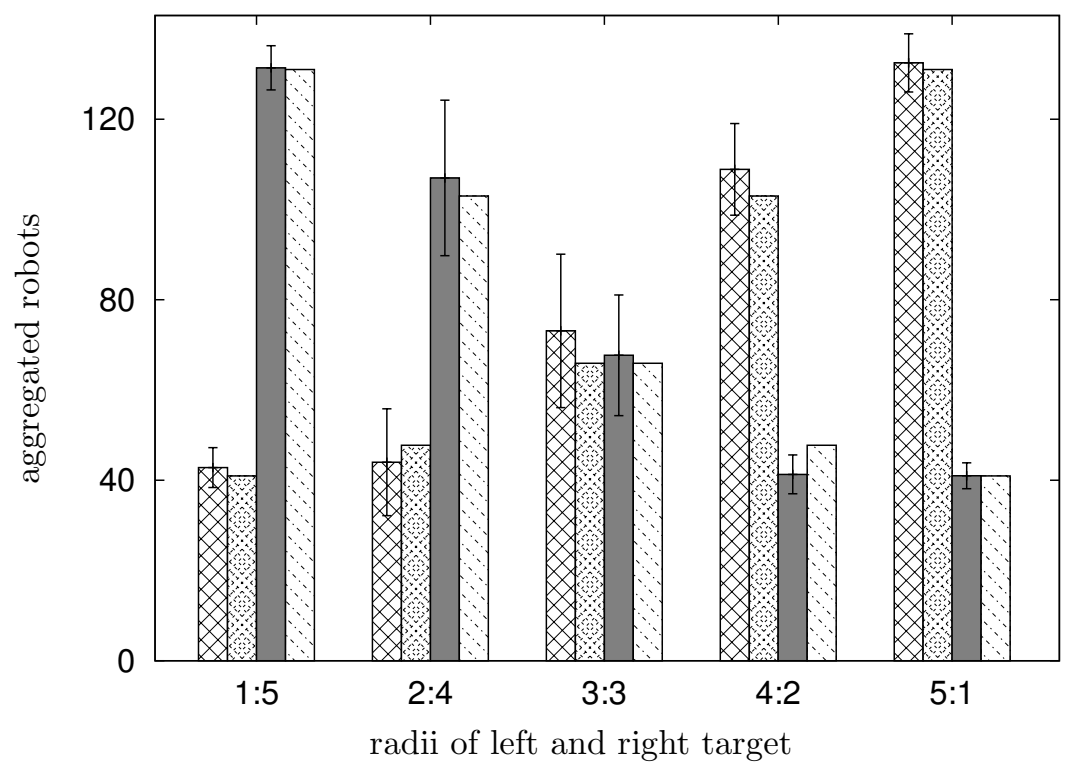

Figure 13: Number of aggregated robots with varied target area radii, comparing the simulation of Schmickl et al. (2007a) to the model: aggregated robots at left target in simulation (1st bar of each cluster) and model (2nd bar), aggregated robots at right target in simulation (3rd bar) and model (4th bar), $\bar{\rho}=0.13$.

were determined by fitting the model to the simulation data of Schmickl et al. (2007a) shown in Fig. 13. The resulting single parameter setting was used to obtain all other results, which will be described in the following section.

\subsubsection{Results}

We verify the model by focusing on three parameters (target radii, average robot density $\bar{\rho}$, and aggregation threshold $\delta_{\text {aggr }}$ ) and by comparing the predictions of our model to the data obtained in simulations reported in (Schmickl et al., 2007a). While the free parameters were fitted to the data of Fig. 13, the other data can definitely be considered as predictions of the model: number of aggregated robots at the target areas depending on the robot density (Fig. 14), number of aggregated robots at the target areas depending on the aggregation threshold $\delta_{\text {aggr }}$ (Fig. 15), and the robot density over space (Fig. 16). Therefore, we have a good fit in Fig. 13 while we have more significant discrepancies in the other figures. The accuracy of these predictions is sufficient such that the model is able to support the algorithm design phase. For example, an appropriate aggregation threshold $\delta_{\text {aggr }}$ can be chosen on the basis of Fig. 15 which shows a balance between occupying mainly the bigger target area and marginally the smaller area.

The systematic deviations in Fig. 14 and 15 (underestimation of aggregated robots at the smaller target area for $0.03<\bar{\rho}<0.2$ and $-200<\delta_{\text {aggr }}<0$ ) seem to be caused by temporal breakdowns of the coherence around the smaller target area. Such breakdowns are more likely for smaller robot densities. Our observations of the simulations show that small, isolated groups of robots are able to establish a potential field with a significant gradient at the smaller target area for a limited time. This gradient suffices to cause a temporary aggregation. Our 


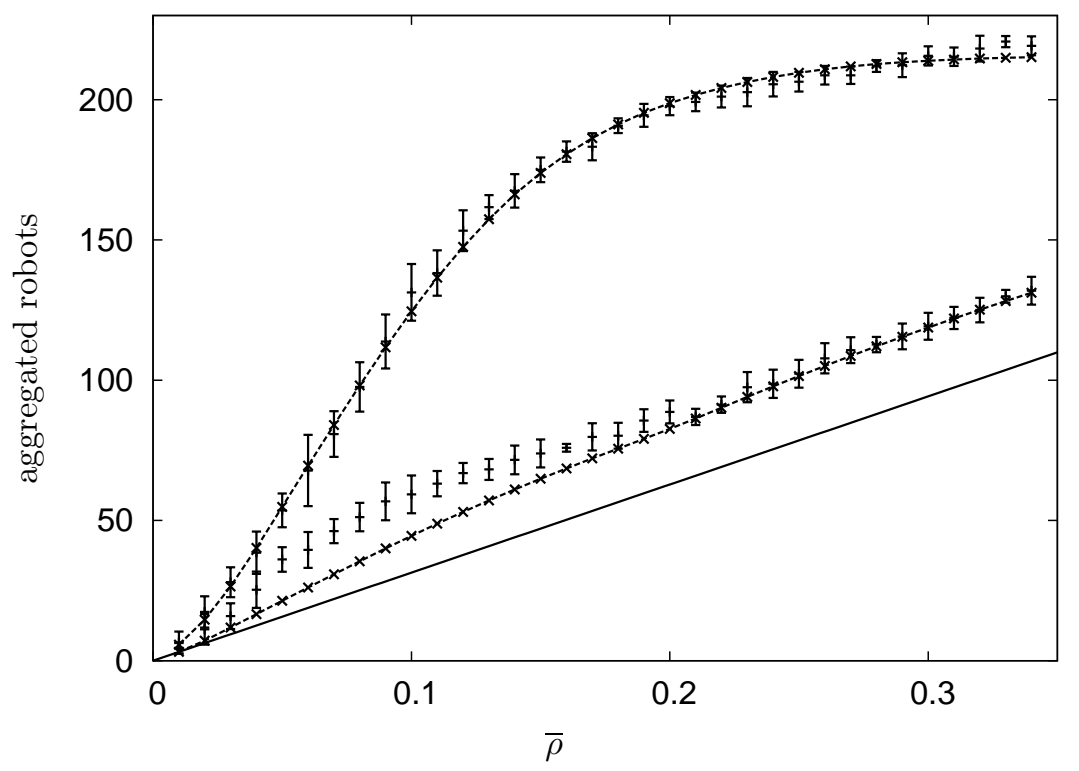

Figure 14: Number of aggregated robots at the small (lower line) and the big target area (upper line) with varied average density $\bar{\rho}$, comparing the model (dashed lines) to the simulation (confidence intervals). The solid line gives the expected number of robots if there is no aggregation, $\delta_{\text {aggr }}=-50$.

approach to compensate such fluctuations with $g_{\text {mod }}$ is not sufficient in the case of smaller robot densities because we averaged over the time by measuring $g_{\text {eff }}$. Thus, for simplicity reasons, we assumed statistical independence over time. However, our observations show a correlation with time. Thus, higher accuracy in the collective perception scenario seems to be impossible based on a Markov approximation. An even more sophisticated approach is apparently needed to overcome this problem.

In Fig. 16 we compare the spatial robot densities of the simulation and the model. The squared error map in Fig. 16(c) indicates an incorrect prediction of the density shape at the bigger target area. This shape is very sensitive to the discount coefficient $d$ (Fig. 11). However, the mean squared error is quite low $(\approx 0.001)$. In addition to the presented examples, we give a generic guideline for modeling robotic swarms using our framework in the following section.

\section{Towards General Methodological Principles}

It seems unlikely that a complete step-by-step manual for designing swarm models independent of the actual application is possible. However, the proposed framework seems to have the potential to be applicable to quite a wide variety of scenarios. In this section we present a few principles for the development of a model based on the Langevin and Fokker-Planck equations. These principles are stated by generically answering three questions which should be asked by the model designer. Still, the model development will remain a partially heuristic process which cannot be generalized for all kinds of application.

The first question is: Are there several states in the controller and should all of them be explicitly modeled? In principle, one has to introduce a Langevin and a Fokker-Planck 


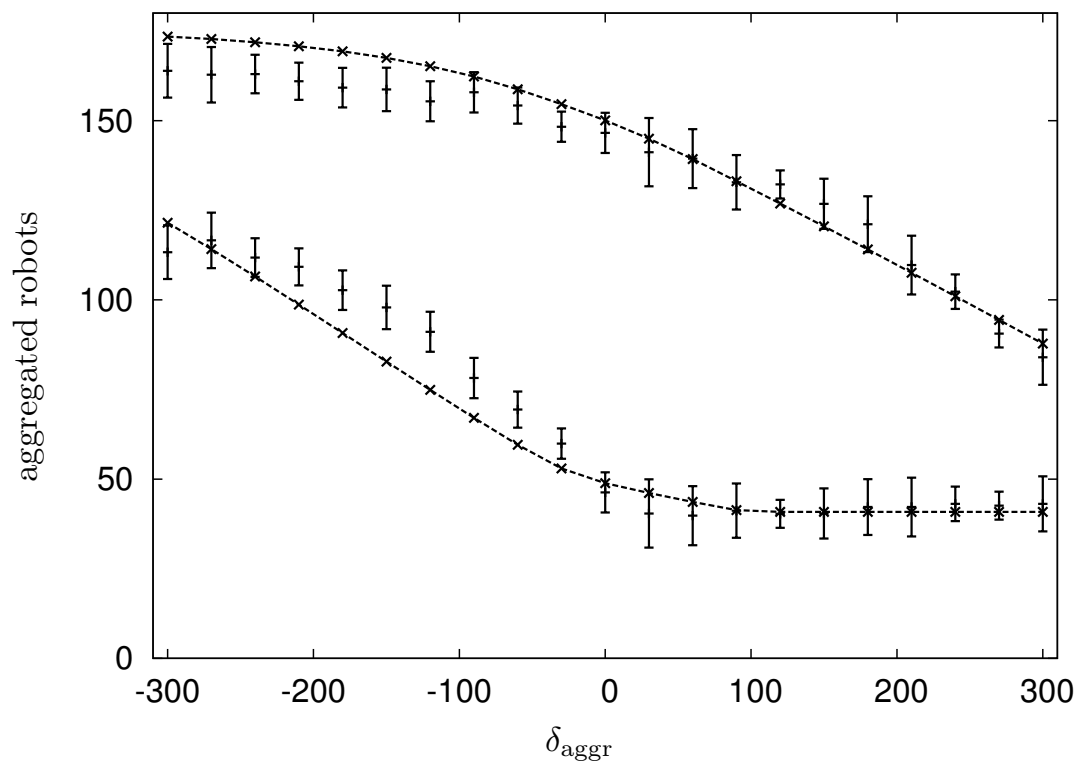

Figure 15: Number of aggregated robots at the small (lower line) and the big target area (upper line) depending on aggregation threshold $\delta_{\text {aggr }}$, comparing the model (dashed lines) to the simulation (confidence intervals).

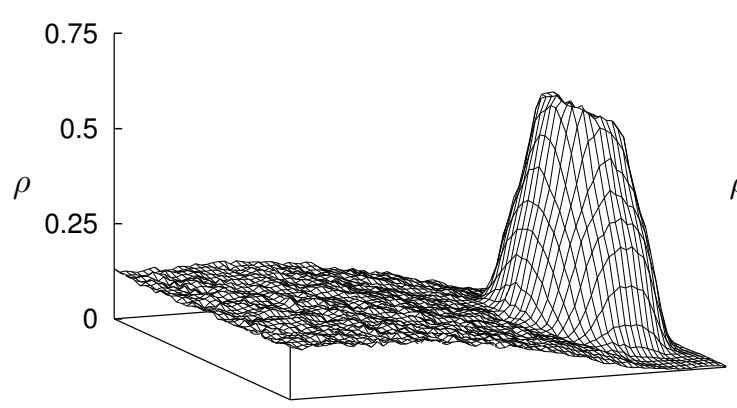

(a) Simulation, mean of 600 samples.

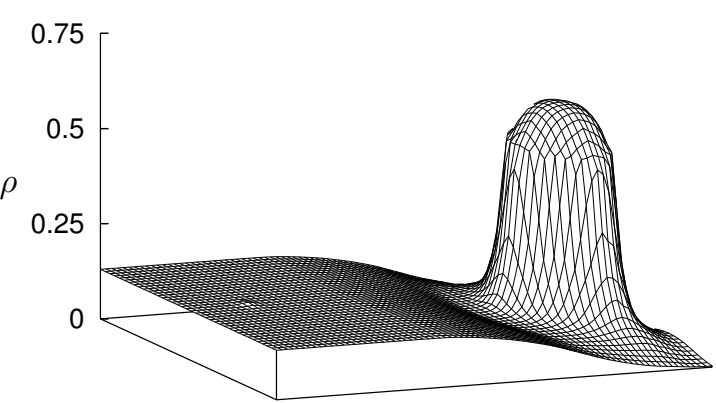

(b) Model.

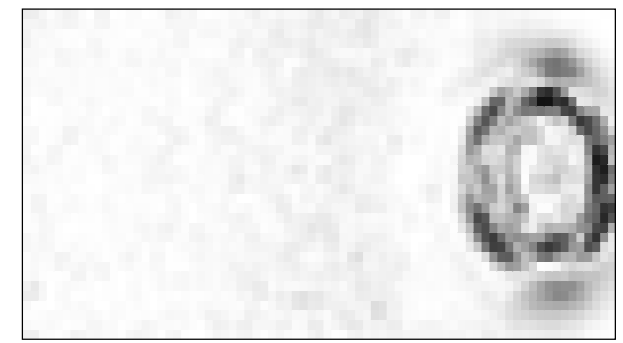

(c) Squared error, errors are linearly mapped to gray values, maximum about 0.035 .

Figure 16: Comparison of the robot density $\rho(\mathbf{r}, t=250)$ between simulation and model. 
equation for each state $m$. Thus, one gets a set of Langevin equations

$$
\dot{\mathbf{R}}(t)=-\mathbf{A}_{m}(\mathbf{R}(t), t)+B_{m}(\mathbf{R}(t), t) F(t)
$$

plus a formalism determining the transitions between states. The corresponding set of FokkerPlanck equations is:

$$
\frac{\partial \rho_{m}}{\partial t}=-\nabla\left(\mathbf{A}_{\mathbf{m}} \rho_{m}\right)+\frac{1}{2} \nabla^{2}\left(B_{m}^{2} \rho_{m}\right)+\sum_{n \neq m} t_{n, m} \rho_{n}-t_{m, n} \rho_{m}
$$

for transition rates $t_{n, m}$ which define the probability of switching from state $n$ to $m$. Note the similarity to the master equation and, hence, the similarity to the rate equation approaches (Lerman et al., 2005) introduced by the sum handling the state switches. For an example of such a multi-state approach see (Hamann and Wörn, 2007a). However, it is not always necessary to model each state explicitly as shown above. The approach presented in the emergent taxis scenario is generally possible, if the differences of the $\mathbf{A}$ - and $B$-functions for each state can be expressed by a spatiotemporal function like the $s$-function in the emergent taxis scenario.

The second question is: When do the robots move randomly and when do they move in a goal-oriented manner? If these ratios are expressible in scalar functions, the ratios are incorporated in $B$ and as a part of $\mathbf{A}$. They might depend on external influences as well, which leads to the third question, which is: Based on what information do the robots move in a goal-oriented manner? The information should be a direction which is incorporated in $\mathbf{A}$. However, it is possible there is only exploration, making $\mathbf{A}$ obsolete. Typically, the information depends on a natural spatiotemporal process. Such processes have models wellknown from physics. If high-level communication between the robots is used one has to introduce empirical parameters and/or functions as discussed above.

\section{Discussion and Conclusion}

Our model framework supports the algorithm design in two ways - directly and indirectly. First, it reduces the number of time-consuming experiments in simulation or with the actual hardware by providing an estimate of the behavior which is to be expected. Second, one might profit from a supposed disadvantage of the model. In contrast to a simulation, our model is only partially defined by microscopic features. Therefore, the user is forced to think about macroscopic effects. One is inspired to do so metaphorically within the theoretical framework of the underlying concepts of the applied equations, for example, diffusion or kinetic theory of gases. Thus, the inspiration from physics helps to understand the macroscopic processes just as the inspiration from biology does to find a general approach.

Another benefit of this model is that some macroscopic aspects can be derived from a microscopic description concerning mainly the robots' motion. However, as we reported in the two examples given above, usually this derivation cannot be done for the complete behavior due to the complexity of such systems. In both cases the macroscopic model was not derived formally. Typically, the communication is problematic and it is very questionable whether macroscopic features can be derived from a microscopic description for algorithms using explicit communication based on non-trivial protocols. In our work we bypass the challenge of modeling communication by introducing empirically-determined parameters and sometimes 
even quite rough approximations. Surely such approximations are undesirable but they only reflect the current state-of-the-art methods. Additionally, the results show the applicability of our approach.

Presumably our model is not fully scalable with respect to the prediction's accuracy. However, this is again a characteristic feature of complex systems. Most likely scalability can only be achieved by switching back to microscopic simulations thereby re-introducing, as a trade-off, the burden of heavy computational intensity.

The application of numerical methods to solve the Fokker-Planck equation generates computational needs as well. Simulation runs are also necessary to measure empirical parameters. However, after having determined the parameters, it takes a few seconds or one minute at most to obtain a quite accurate description of the average behavior. A simulation might run for hours or even days to get statistically significant results, although the achievable accuracy is higher in this case.

Finally, we want to emphasize that the need to determine a parameter setting in swarm robotics is neither an uncommon situation nor an easy task. Typically, the control algorithms have critical parameters with very sensitive effects for the swarm behavior. For example, shifting the $a$-function in the emergent taxis scenario influences whether the swarm moves or does not move at all. Similarly, the aggregation threshold $\delta_{\text {aggr }}$ of the trophallaxis strategy determines whether a discrimination of the smaller target occurs. Thus, determining parameter settings in swarm robotics should not be considered to be merely a fine adjustment but should rather be seen as a partial definition of the behavior. To conclude, we want to emphasize the versatility of our model framework. Qualitatively correct results are easily obtained. Conversely, it is possible to achieve quantitative correctness albeit at higher costs. In future work we intend to verify our model in several scenarios using real hardware.

\section{Acknowledgments}

The authors thank Thomas Schmickl and Christoph Möslinger for providing the data of their simulation runs and the simulation itself. We thank the anonymous referees, the editors, and Sebastian Schuster for many helpful comments as well as Elva Hohe and Christina Meyer for proofreading the manuscript.

Hamann is supported by the German Research Foundation (DFG) within the Research Training Group GRK 1194 Self-organizing Sensor-Actuator Networks.

\section{A Derivation of the Fokker-Planck Equation Based on the Langevin Equation}

We derive the Fokker-Planck equation following closely the derivation of Haken (1977). We restrict ourselves to the derivation of the one-dimensional space variant. The generalization to the two-dimensional case (actually to any dimension) uses the same methods but would require a much longer derivation. Nevertheless, we will discuss some selected issues of the two-dimensional case at the end of this appendix.

We begin by considering the trajectory in one-dimensional space of a Brownian particle in a potential. We describe this trajectory with a probability distribution using the Dirac delta function. The probability distribution of trajectory 1 is 


$$
P_{1}(r, t)=\delta\left(r-r_{1}(t)\right),
$$

for trajectory 2

$$
P_{2}(r, t)=\delta\left(r-r_{2}(t)\right),
$$

and so on. Now, we average all such trajectories and introduce function

$$
\rho(r, t)=\langle P(r, t)\rangle .
$$

Say $p_{i}$ is the probability of trajectory $i$ then the probability distribution can be calculated by evaluating

$$
\rho(r, t)=\sum_{i} p_{i} \delta\left(r-r_{i}(t)\right) .
$$

Using equations 23,24 , and 25 we can rewrite this and get

$$
\rho(r, t)=\langle\delta(r-r(t))\rangle .
$$

The product $\rho(r, t) d r$ is the probability of encountering the particle at position $r$ within the interval defined by $d r$ at time $t$. However, we would need to consider the whole time interval to evaluate equation 26 since the particle could approach position $r$ at any time $t$. To avoid that, we derive a difference equation for $\rho$ by considering the change of $\rho$ within a time interval $\Delta t$

$$
\Delta \rho(r, t) \equiv \rho(r, t+\Delta t)-\rho(r, t)
$$

and using equation 27 we get

$$
\Delta \rho(r, t) \equiv\langle\delta(r-r(t+\Delta t))\rangle-\langle\delta(r-r(t))\rangle .
$$

We set

$$
r(t+\Delta t)=r(t)+\Delta r(t)
$$

and derive the Taylor series till the second power for

$$
\langle\delta(r-r(t)-\Delta r(t))\rangle=\langle\delta(r-r(t))\rangle+\left\langle\left[-\frac{d}{d r} \delta(r-r(t))\right] \Delta r(t)\right\rangle+\frac{1}{2}\left\langle\left[\frac{d^{2}}{d r^{2}} \delta(r-r(t))\right](\Delta r(t))^{2}\right\rangle .
$$

Applying this Taylor series to equation 29 results in

$$
\Delta \rho(r, t)=\left\langle\left[-\frac{d}{d r} \delta(r-r(t))\right] \Delta r(t)\right\rangle+\frac{1}{2}\left\langle\left[\frac{d^{2}}{d r^{2}} \delta(r-r(t))\right](\Delta r(t))^{2}\right\rangle .
$$

Now, we are in a position to draw the relation between this derivation of the Fokker-Planck equation and the underlying Langevin equation

$$
\dot{R}(t)=-A(R(t), t)+B(R(t), t) F(t)
$$


that helps us to calculate $\Delta r$ by integration. We assume that the changes of $r, A$, and $B$ are small within $\Delta t$ and that already many collisions have occurred. Thus, we can approximate $\int_{t}^{t+\Delta t} r\left(t^{\prime}\right) d t^{\prime} \approx r(t) \Delta t, \int_{t}^{t+\Delta t} A\left(r\left(t^{\prime}\right), t^{\prime}\right) d t^{\prime} \approx A(r(t), t) \Delta t$, and $B(r(t), t) \approx B(r(t+\Delta t), t+$ $\Delta t)$. We get

$$
\begin{aligned}
\int_{t}^{t+\Delta t} \dot{r}\left(t^{\prime}\right) d t^{\prime} & =r(t+\Delta t)-r(t) \equiv \Delta r \\
& =-\int_{t}^{t+\Delta t} A\left(r\left(t^{\prime}\right), t^{\prime}\right) d t^{\prime}+\int_{t}^{t+\Delta t} B(r(t), t) F\left(t^{\prime}\right) d t^{\prime}=-A(r(t), t) \Delta t+B(r(t), t) \Delta F(t) .
\end{aligned}
$$

Putting the right hand side of equation 34 in the first term of the right hand side of equation 32 results in

$$
\frac{d}{d r}[\langle\delta(r-r(t))(-A(r(t), t) \Delta t)\rangle+\langle\delta(r-r(t)) B(r(t), t)\rangle\langle\Delta F\rangle] .
$$

Separating $\langle\Delta F\rangle$ is approved since $\Delta F$ contains all collisions after $t$, but $B(r(t), t)$ and $r(t)$ are determined by all collisions before $t$, and the collisions themselves are independent from each other. Thus, the overall mean value can be expressed as a product of mean values. We assume $\Delta F$ and $B$ being uncorrelated and substitute $r(t)$ by $r$ (see Haken (1977, chap. 6) for details). The average of $\Delta F$ vanishes because the average of $F$ vanishes. Hence, equation 36 is reduced to

$$
\Delta t \frac{d}{d r}[\langle\delta(r-r(t)) A(r, t)\rangle]
$$

Using the same arguments as before we can restate the second term of the right hand side of equation 32

$$
\frac{d^{2}}{d r^{2}}\left[\langle\delta(r-r(t))\rangle\left\langle(\Delta r)^{2}(t)\right\rangle\right] .
$$

Substituting $\Delta r$ by the right hand side of equation 34 results in terms containing $\Delta F, \Delta F \Delta t$, or $(\Delta t)^{2}$. The terms with $\Delta F$ vanish. The product including $(\Delta F)^{2}$ is the only non-zero term being linear in $\Delta t$. We get

$$
\left\langle(\Delta F)^{2}\right\rangle \equiv\langle\Delta F(t) \Delta F(t)\rangle=\int_{t}^{t+\Delta t} \int_{t}^{t+\Delta t}\left\langle F\left(t^{\prime}\right) F\left(t^{\prime \prime}\right)\right\rangle d t^{\prime} d t^{\prime \prime} .
$$

Assuming a $\delta$-correlation for $F$

$$
\left\langle\Delta F(t) \Delta F\left(t^{\prime}\right)\right\rangle=Q \delta\left(t-t^{\prime}\right)
$$

we are in a position to evaluate equation 39 and we get

$$
Q \Delta t
$$

Thus, we end up with a new form for equation 38 


$$
\frac{d^{2}}{d r^{2}}\left[\left\langle\delta(r-r(t)) B^{2}(r, t)\right\rangle Q \Delta t\right] .
$$

Dividing the original equation 32 by $\Delta t$ and using the two results (42 and 37) we get

$$
\frac{d \rho(r, t)}{d t}=-\frac{d}{d r}(A(r(t), t) \rho(r, t))+\frac{1}{2} Q \frac{d^{2}}{d r^{2}}\left(B^{2}(r(t), t) \rho(r, t)\right),
$$

which is the Fokker-Planck equation describing the temporal evolution of a particle's probability distribution in one-dimensional space. As mentioned above, the two-dimensional case is derived using the same methods. However, a tight representation of this derivation is hardly possible. The two-dimensional equivalent of equation 27 is

$$
\rho(\mathbf{r}, t)=\left\langle\delta^{2}(\mathbf{r}-\mathbf{r}(t))\right\rangle,
$$

with $\delta^{2}(\mathbf{r})=\delta\left(r_{x}\right) \delta\left(r_{y}\right)$. Then for the Taylor series (cf. eq. 31) we get

$$
\begin{aligned}
\left\langle\delta^{2}(\mathbf{r}-\mathbf{r}(t)-\Delta \mathbf{r}(t))\right\rangle= & \left\langle\delta^{2}(\mathbf{r}-\mathbf{r}(t))\right\rangle+\left\langle(\Delta \mathbf{r}(t) \cdot \nabla) \delta^{2}(\mathbf{r}-\mathbf{r}(t))\right\rangle \\
& +\frac{1}{2}\left\langle\Delta \mathbf{r}(t) \cdot\left[\Delta \mathbf{r}(t) \cdot \nabla\left(\nabla \delta^{2}(\mathbf{r}-\mathbf{r}(t))\right)\right]\right\rangle
\end{aligned}
$$

and the two-dimensional Langevin equation equivalent to equation 33, which is used throughout this paper, is

$$
\dot{\mathbf{R}}(t)=-\mathbf{A}(\mathbf{R}(t), t)+B(\mathbf{R}(t), t) \mathbf{F}(t) .
$$

Concerning the correlations of $(\Delta \mathbf{F})^{2}$ (cf. eq. 40) all combinations of the components need to be considered in general

$$
\begin{aligned}
\left\langle\Delta F_{x}(t) \Delta F_{x}\left(t^{\prime}\right)\right\rangle & =Q_{x x} \delta\left(t-t^{\prime}\right), \\
\left.\left\langle\Delta F_{x}(t) \Delta F_{y}\left(t^{\prime}\right)\right\rangle=\Delta F_{y}(t) \Delta F_{x}\left(t^{\prime}\right)\right\rangle=Q_{x y} \delta\left(t-t^{\prime}\right) & =Q_{y x} \delta\left(t-t^{\prime}\right), \\
\left\langle\Delta F_{y}(t) \Delta F_{y}\left(t^{\prime}\right)\right\rangle & =Q_{y y} \delta\left(t-t^{\prime}\right) .
\end{aligned}
$$

However, in this work the $x$ - and $y$-components are considered being uncorrelated $\left(Q_{y x}=\right.$ $\left.Q_{x y}=0\right)$ and we set $Q=Q_{x x}=Q_{y y}$. Thus, we get

$$
\frac{\partial \rho(\mathbf{r}, t)}{\partial t}=-\nabla(\mathbf{A}(\mathbf{r}(t), t) \rho(\mathbf{r}, t))+\frac{1}{2} Q \nabla^{2}\left(B^{2}(\mathbf{r}(t), t) \rho(\mathbf{r}, t)\right),
$$

where $\rho(\mathbf{r}, t) d r_{x} d r_{y}$ is the probability of encountering the particle at position $\mathbf{r}$ within the rectangle defined by $d r_{x}$ and $d r_{y}$ at time $t$.

\section{B Numerics of the Fokker-Planck Equation}

The Fokker-Planck equation is a partial differential equations (PDE) and cannot be solved explicitly except for a few special cases which are of no interest in this work (Risken, 1984; van Kampen, 1981). Therefore, we need to apply methods of numerical integration. 


\section{B.1 Discretizing}

Before methods of numerical integration can be applied to solve the Fokker-Planck equation, we need to discretize the equation. The three most important methods are: finite differences, finite volumes, and finite elements. Here, we use the finite difference method. Time is transformed to discrete time steps and space is discretized by a finite grid.

The following three differences are the building blocks for discretizing the Fokker-Planck equation: the first-order forward difference for the time derivative at time $n$ and point $\xi$ in one-dimensional space

$$
\frac{\partial u_{\xi}^{n}}{\partial t} \doteq \frac{u_{\xi}^{n+1}-u_{\xi}^{n}}{\Delta t}
$$

the first-order central difference for the space derivative

$$
\frac{\partial u_{\xi}^{n}}{\partial x} \doteq \frac{u_{\xi+1}^{n}-u_{\xi-1}^{n}}{2 \Delta x} ;
$$

and the second-order central difference for the space derivative

$$
\frac{\partial\left(u_{\xi}^{n}\right)^{2}}{\partial x^{2}} \doteq \frac{u_{\xi+1}^{n}-2 u_{\xi}^{n}+u_{\xi-1}^{n}}{(\Delta x)^{2}} .
$$

Once the equation is discretized we transform it to a linear system of equations and then need an efficient method to solve it. This is discussed in the next section.

\section{B.2 Solving}

In the following we restrict ourselves to the treatment of initial value problems only. That is, we consider problems with given values for one point in time and we are interested in the evolution forward in time. The simplest way of solving the discretized equation is the forward integration in time. This method just implements the finite differences directly by summing over the time steps.

However, this method has a poor stability, that is, errors due to the limited accuracy of the floating point arithmetic might successively be magnified. Instability is one of the main issues in numerics (Higham, 2002). To overcome the problem of instability in case of the forward integration only small step sizes in space and time are allowed. However, this makes many iterations necessary to calculate the evolution in time.

Fortunately, numerics provides us with more sophisticated methods to speed this process up and to stay stable. We use the Crank-Nicholson method (Crank and Nicolson, 1947) combined with the alternate-direction implicit method (ADI). The Crank-Nicholson method is based on the central difference in space, and the trapezoidal rule in time (i.e., it is implicit). A numerical method is implicit, if the value being determined occurs also on the right hand side of the equation. The Crank-Nicholson method for two dimensions in space and time step size of $\Delta t$

$$
u_{j, k}^{n+1}=u_{j, k}^{n}+\frac{\Delta t}{2}\left(Q_{j, k}^{n+1}+Q_{j, k}^{n}\right),
$$

with $Q_{j}^{n}$ is the discretization of the PDE's right hand side or of a single operator respectively, for example in case of the two-dimensional diffusion equation

$$
\frac{\partial u}{\partial t}=D\left(\frac{\partial^{2} u}{\partial x^{2}}+\frac{\partial^{2} u}{\partial y^{2}}\right),
$$


we get in a uniform grid with grid point distances of $\Delta x$

$$
Q_{j, k}^{n}=\frac{D}{(\Delta x)^{2}}\left(u_{j+1, k}^{n}+u_{j-1, k}^{n}+u_{j, k+1}^{n}+u_{j, k-1}^{n}-4 u_{j, k}^{n}\right) .
$$

Equation 55 with $Q$ defined by equation 57 written in matrix notation results in a matrix with five non-zero diagonals. Less non-zero diagonals would be desirable, for example, a tridiagonal matrix is disproportionately easier to solve.

This can be achieved by ADI which is an operator splitting method reducing the system of equations to an easier to solve problem. For example, the two-dimensional diffusion equation splits into two equations

$$
\begin{aligned}
& u_{j, k}^{n+\frac{1}{2}}=u_{j, k}^{n}+\frac{D \Delta t}{2(\Delta x)^{2}}\left(u_{j+1, k}^{n+\frac{1}{2}}-2 u_{j, k}^{n+\frac{1}{2}}+u_{j-1, k}^{n+\frac{1}{2}}+u_{j, k+1}^{n}-2 u_{j, k}^{n}+u_{j, k-1}^{n}\right), \\
& u_{j, k}^{n+1}=u_{j, k}^{n+\frac{1}{2}}+\frac{D \Delta t}{2(\Delta x)^{2}}\left(u_{j+1, k}^{n+\frac{1}{2}}-2 u_{j, k}^{n+\frac{1}{2}}+u_{j-1, k}^{n+\frac{1}{2}}+u_{j, k+1}^{n+1}-2 u_{j, k}^{n+1}+u_{j, k-1}^{n+1}\right) .
\end{aligned}
$$

Finally, we have to define the boundary conditions and the initial values of the grid for $t=0$. Here, we have Neumann boundary conditions, that is, they are defined by the derivative on the boundary of the domain. For the case of a totally isolated boundary (i.e., no agent will ever leave the domain) we set

$$
\frac{\partial u}{\partial \nu}(\mathbf{x})=0, \quad \forall \mathbf{x} \in \partial \Omega,
$$

for boundary $\Omega \subset \mathbb{R}^{2}$ and $\nu$ denotes the normal to the boundary. Then we get, for example, for equation 58 and a grid point $(j, K)$ at an outest column $K$

$$
u_{j, K}^{n+\frac{1}{2}}=u_{j, K}^{n}+\frac{D \Delta t}{2(\Delta x)^{2}}\left(u_{j+1, K}^{n+\frac{1}{2}}-2 u_{j, K}^{n+\frac{1}{2}}+u_{j-1, K}^{n+\frac{1}{2}}-u_{j, K}^{n}+u_{j, K-1}^{n}\right) .
$$

The system of equations defined by equations 58-61 can simply be expressed using tridiagonal matrices (the values of the grid points are represented by a single, huge vector $\mathbf{u}$ )

$$
\mathbf{M u}^{n+1}=\mathbf{N u}^{n}
$$

with

$$
\mathbf{M}=\left(\begin{array}{cccccccccc}
-1 & 1 & & & & & & & \cdots & 0 \\
1 & -2 & 1 & & & & & & & \vdots \\
& & \ddots & & & & & & & \\
& & 1 & -2 & 1 & & & & & \\
& & & 1 & -1 & & & & & \\
& & & & & -1 & 1 & & & \\
& & & & & 1 & -2 & 1 & & \\
\vdots & & & & & & & & & \\
0 & & & & & & & 1 & -2 & 1 \\
& & & & & & & 1 & -1
\end{array}\right)
$$


and $\mathbf{N}$ accordingly. For a $j \times k$ grid we have $j k$ unknowns and $j k$ equations. By defining the initial values of the grid $u_{j, k}^{0}$ we complete the mathematical description of the initial value problem and can start to solve it.

The linear system of equation 62 is represented by sparse matrices in the computer. It is solved by fast iterative solvers such as Jacobi, Gauss-Seidel, successive over-relaxation, or conjugate gradients. We do not specify any of these methods since, once we have reduced the original equation to a linear system of equations, several out-of-the-box tools (e.g., Matlab ${ }^{\mathrm{TM}}$, $\mathrm{Maple}^{\mathrm{TM}}$ ) can be used to solve it. However, these standard tools do not provide an automatic discretization of non-standard PDE depending not only on constant coefficients but might even be coupled to other PDE.

\section{References}

Bettstetter, C. (2004). On the connectivity of Ad Hoc networks. The Computer Journal, $47(4): 432-447$.

Bjerknes, J. D., Winfield, A., and Melhuish, C. (2007). An analysis of emergent taxis in a wireless connected swarm of mobile robots. In IEEE Swarm Intelligence Symposium, pages 45-52, Los Alamitos, CA. IEEE Press.

Brown, R. (1828). A brief account of microscopical observations made in the months of June, July and August, 1827, on the particles contained in the pollen of plants; and on the general existence of active molecules in organic and inorganic bodies. Philosophical Magazine, 4:161-173.

Correll, N. (2007). Coordination schemes for distributed boundary coverage with a swarm of miniature robots: synthesis, analysis and experimental validation. PhD thesis, Ecole Polytechnique Fédérale de Lausanne.

Correll, N. and Martinoli, A. (2006). System identification of self-organizing robotic swarms. In Gini, M. and Voyles, R., editors, Proceedings of the 8th Int. Symp. on Distributed Autonomous Robotic Systems (DARS'06), pages 31-40, Berlin, Germany. Springer-Verlag.

Crank, J. and Nicolson, P. (1947). A practical method for numerical evaluation of solutions of partial differential equations of the heat conduction type. In Proceedings of the Cambridge Philosophical Society, volume 43, pages 50-64.

Deguet, J., Demazeau, Y., and Magnin, L. (2006). Elements about the emergence issue: A survey of emergence definitions. Complexus, 3(1-3):24-31.

Doob, J. L. (1953). Stochastic Processes. Wiley, New York.

Edelstein-Keshet, L. (2006). Mathematical models of swarming and social aggregation. Robotica, 24(3):315-324.

Einstein, A. (1905). Über die von der molekularkinetischen Theorie der Wärme geforderte Bewegung von in ruhenden Flüssigkeiten suspendierten Teilchen. Annalen der Physik, $17: 549-560$. 
Feddema, J. T., Lewis, C., and Schoenwald, D. A. (2002). Decentralized control of cooperative robotic vehicles: theory and application. IEEE Transactions on Robotics and Automation, $18(5): 852-864$.

Fokker, A. D. (1914). Die mittlere Energie rotierender elektrischer Dipole im Strahlungsfeld. Annalen der Physik, 348(5):810-820.

Galstyan, A., Hogg, T., and Lerman, K. (2005). Modeling and mathematical analysis of swarms of microscopic robots. In Proceedings of IEEE Swarm Intelligence Symposium (SIS'05), pages 201-208, Los Alamitos, CA. IEEE Press.

Gazi, V. and Passino, K. M. (2003). Stability analysis of swarms. IEEE Transactions on Automatic Control, 48(4):692-697.

Grünbaum, D. and Okubo, A. (1994). Modeling social animal aggregations. Frontiers in Theoretical Biology, 100:296-325.

Haken, H. (1977). Synergetics - an introduction. Springer-Verlag, Berlin, Germany.

Hamann, H. and Wörn, H. (2007a). An analytical and spatial model of foraging in a swarm of robots. In Şahin, E., Spears, W., and Winfield, A. F., editors, Swarm Robotics - Second $S A B 2006$ International Workshop, volume 4433 of LNCS, pages 43-55, Berlin, Germany. Springer-Verlag.

Hamann, H. and Wörn, H. (2007b). A space- and time-continuous model of self-organizing robot swarms for design support. In First IEEE International Conference on Self-Adaptive and Self-Organizing Systems (SASO'07), pages 23-31, Los Alamitos, CA. IEEE Press.

Helbing, D., Schweitzer, F., Keltsch, J., and Molnar, P. (1997). Active walker model for the formation of human and animal trail systems. Physical Review E, 56(3):2527-2539.

Higham, N. J. (2002). Accuracy and Stability of Numerical Algorithms. Society for Industrial and Applied Mathematics.

Hogg, T. (2006). Coordinating microscopic robots in viscous fluids. Autonomous Agents and Mutli-Agent Systems, 14(3):271-305.

Holland, J. H. (1998). Emergence - From Chaos to Order. Oxford University Press, New York.

Kolmogorov, A. N. (1931). Über die analytischen Methoden in der Wahrscheinlichkeitsrechnung. Mathematische Annalen, 104(1):415-458.

Langevin, P. (1908). Sur la théorie du mouvement brownien. Comptes-rendus de l'Académie des Sciences, 146:530-532.

Langton, C. G., editor (1989). Artificial Life: Proceedings of an Interdisciplinary Workshop on the Synthesis and Simulation of Living Systems, Reading, MA. Addison-Wesley.

Lemons, D. S. and Gythiel, A. (1997). Paul langevin's 1908 paper "On the theory of Brownian motion" "Sur la théorie du mouvement brownien," Comptes-rendus de l'Académie des Sciences (Paris) 146, 530-533 (1908)]. American Journal of Physics, 65(11):1079-1081. 
Lerman, K., Martinoli, A., and Galstyan, A. (2005). A review of probabilistic macroscopic models for swarm robotic systems. In Şahin, E. and Spears, W. M., editors, Swarm Robotics - SAB 2004 International Workshop, pages 143-152, Berlin, Germany. Springer-Verlag.

Martinoli, A. (1999). Swarm Intelligence in Autonomous Collective Robotics: From Tools to the Analysis and Synthesis of Distributed Control Strategies. PhD thesis, Ecole Polytechnique Fédérale de Lausanne.

Martinoli, A., Easton, K., and Agassounon, W. (2004). Modeling swarm robotic systems: A case study in collaborative distributed manipulation. Int. Journal of Robotics Research, 23(4):415-436.

McCanne, S., Floyd, S., Fall, K., Varadhan, K., et al. (1997). Network simulator - ns-2. http://www-mash.cs.berkeley.edu/ns/.

Mogilner, A. and Edelstein-Keshet, L. (1999). A non-local model for a swarm. Journal of Mathematical Biology, 38(6):534-570.

Nembrini, J., Winfield, A. F., and Melhuish, C. (2002). Minimalist coherent swarming of wireless networked autonomous mobile robots. In Hallam, B., Floreano, D., Hallam, J., Hayes, G., and Meyer, J.-A., editors, Proceedings of the seventh international conference on simulation of adaptive behavior (From animals to animats), pages 373-382, Cambridge, MA. MIT Press.

Neumann, J. v. (1966). The Theory of Self-Reproducing Automata. Arthur Burks (ed.), University of Illinois Press, Champaign, IL.

Okubo, A. (1986). Dynamical aspects of animal grouping: Swarms, schools, flocks, and herds. Advances in Biophysics, 22:1-94.

Okubo, A. and Levin, S. A. (2001). Diffusion and Ecological Problems: Modern Perspectives. Springer-Verlag, Berlin, Germany.

Planck, M. (1917). Über einen Satz der statistischen Dynamik and seine Erweiterung in der Quantentheorie. Sitzungsberichte der Preußischen Akademie der Wissenschaften, 24(324$341)$.

Risken, H. (1984). The Fokker-Planck Equation. Springer-Verlag, Berlin, Germany.

Schillo, M., Fischer, K., and Klein, C. T. (2000). The micro-macro link in DAI and sociology. In Moss, S. and Davidsson, P., editors, Multi-Agent-Based Simulation: Second International Workshop, (MABS'00), volume 1979 of LNCS, pages 303-317, Berlin, Germany. SpringerVerlag.

Schmickl, T. and Crailsheim, K. (2006). Trophallaxis among swarm-robots: A biologically inspired strategy for swarm robotics. In The First IEEE/RAS-EMBS International Conference on Biomedical Robotics and Biomechatronics (BioRob'06), pages 377-382, Los Alamitos, CA. IEEE Press.

Schmickl, T. and Crailsheim, K. (2008). Trophallaxis within a robotic swarm: bio-inspired communication among robots in a swarm. Autonomous Robots, 25(1-2):171-188. 
Schmickl, T., Möslinger, C., and Crailsheim, K. (2007a). Collective perception in a robot swarm. In Şahin, E., Spears, W. M., and Winfield, A. F., editors, Swarm Robotics - Second $S A B 2006$ International Workshop, volume 4433 of $L N C S$, pages 144-157, Berlin, Germany. Springer-Verlag.

Schmickl, T., Möslinger, C., Thenius, R., and Crailsheim, K. (2007b). Bio-inspired navigation of autonomous robots in heterogenous environments. International Journal of Factory Automation, Robotics and Soft Computing, 3:164-170.

Schmickl, T., Möslinger, C., Thenius, R., and Crailsheim, K. (2007c). Individual adaptation allows collective path-finding in a robotic swarm. International Journal of Factory Automation, Robotics and Soft Computing, 4:102-108.

Schweitzer, F. (2003). Brownian Agents and Active Particles. On the Emergence of Complex Behavior in the Natural and Social Sciences. Springer-Verlag, Berlin, Germany.

Smoluchowski, M. v. (1906). Zur kinetischen Theorie der Brownschen Molekularbewegung und der Suspensionen. Annalen der Physik, 21:756-780.

Soysal, O. and Şahin, E. (2007). A macroscopic model for self-organized aggregation in swarm robotic systems. In Şahin, E., Spears, W. M., and Winfield, A. F., editors, Swarm Robotics - Second SAB 2006 International Workshop, volume 4433 of LNCS, pages 27-42, Berlin, Germany. Springer-Verlag.

van Kampen, N. G. (1981). Stochastic processes in physics and chemistry. North-Holland, Amsterdam.

Vicsek, T., Czirok, A., Ben-Jacob, E., Cohen, I., and Shochet, O. (1995). Novel type of phase transition in a system of self-driven particles. Physical Review Letters, 6(75):1226-1229.

Winfield, A. F., Sav, J., Fernández-Gago, M.-C., Dixon, C., and Fisher, M. (2005). On formal specification of emergent behaviours in swarm robotic systems. International Journal of Advanced Robotic Systems, 2(4):363-370.

Yamins, D. (2005). Towards a theory of "local to global" in distributed multi-agent systems. In Proceedings of the fourth international joint conference on Autonomous agents and multiagent systems (AAMS'05), pages 183 - 190, New York, NY. ACM. 